\title{
GENERALIZED QUANTIFIERS AND COMPACT LOGIC
}

\author{
BY \\ SAHARON SHELAH
}

ABSTRACT. We solve a problem of Friedman by showing the existence of a logic stronger than first-order logic even for countable models, but still satisfying the general compactness theorem, assuming e.g. the existence of a weakly compact cardinal. We also discuss several kinds of generalized quantifiers.

Introduction. We assume the reader is acquainted with Lindström's articles [Li 1] and [Li 2] where he defined "abstract logic" and showed in this framework simple characterizations of first-order logic. For example, it is the only logic satisfying the compactness theorem and the downward Löwenheim-Skolem theorem. Later this was rediscovered by Friedman [Fr 1]; and Barwise [Ba 1] dealt with characterization of infinitary languages.

Keisler asked the following question:

(1) Is there a compact logic (i.e., a logic satisfying the compactness theorem) stronger than first-order logic? It should be mentioned that it is known for many $L\left(Q_{\aleph_{\alpha}}\right)$ that they satisfy the $\lambda$-compactness theorem for $\lambda<\aleph_{\alpha}$ (for $\alpha>0$ ). $\left(Q_{\aleph_{\alpha}}(x) \Leftrightarrow\right.$ there are $\geqslant \aleph_{\alpha} x$ 's; the $\lambda$-compactness theorem says that if $T$ is a theory in $L\left(Q_{\aleph_{\alpha}}\right),|T| \leqslant \lambda$, and for all finite $t \subseteq T$ there is a model, then $T$ has a model.) For example, this is the case for $\alpha=1$. See Fuhrken [Fu 1], Keisler [Ke 2] and see [CK] for general information.

At the Cambridge summer conference of 1971 Friedman asked:

(2) Is there a logic satisfying the compactness theorem, or even the $\aleph_{0}$ compactness theorem, which is stronger than first-order logic even for countable models, i.e., is there a sentence $\psi$ in the logic such that there is no first order sentence $\varphi$ such that for all countable models $M, M \vDash \psi \Longleftrightarrow M \vDash \varphi$ ?

Notice that the power quantifiers $Q_{\aleph_{\alpha}}$ do not satisfy the second part of (2). The quantifier saying " $\varphi(x, y)$ is an ordering with cofinality $\aleph_{1}$ " solves (1) (but obviously not (2)) as proved, in fact in [Sh $2, \S 4.4]$ and noticed by me in Cambridge.

The main result of this paper is the presentation in $\S 1$ of an example solving both (1) and (2) positively (assuming the existence of a weakly compact cardinal); thus, compactness alone does not characterize first-order logic. In $\$ 2$ we mention 
all kinds of problems about generalized second-order quantifiers, and prove some results.

After the solution Friedman asked:

(3) Is there a compact logic, stronger than first-order logic even for finite models?

Notation. $\lambda, \mu, \kappa, \chi$ designate cardinals; $i, j, k, l, \alpha, \beta, \gamma, \delta, \xi$ designate ordinals; and $m, n$ are natural numbers. The power of $A$ is $|A|$. Models are $M, N$, and the universe of $M$ is $|M| . a, b, c$ are elements; $\bar{a}, \bar{b}, \bar{c}$ finite sequences of elements; $l(\bar{a})$ is the length of the sequence $\bar{a} . x, y, z, v$ will be variables, and $\bar{x}, \bar{y}, \bar{z}, \bar{v}$ sequences of variables.

1. A compact logic different from first-order logic. The following theorem is proven under the assumption of the existence of a weakly compact cardinal (see Silver [Si 1]).

THEOREM 1.1. (There is a weakly compact cardinal $\kappa$.) There is a compact logic $L^{*}$, which is stronger than first-order logic even for countable models.

Definition 1.1. $\operatorname{cf}(A,<)$, the cofinality of the ordering $<$ on the set $A$, is the first cardinal $\lambda$ such that there exists $B \subseteq A,|B|=\lambda, B$ is unbounded from above in $A . \operatorname{cf}^{*}(A,<)$ is $\operatorname{cf}(A,>),>$ the reverse order. When $<$ is understood we just write $\operatorname{cf}(A)$ or $\operatorname{cf}^{*}(A)$. It is easy to see that the cofinality is a regular cardinal (or 0 or 1 ).

Definition 1.2. $\left(A_{1}, A_{2}\right)$ is a Dedekind cut of the ordered set $(A,<)$ (or just cut for short) if $A_{1} \cup A_{2}=A ; b_{1} \in A_{1} \wedge b_{2} \in A_{2} \rightarrow b_{1}<b_{2} ; b<$ $b_{1} \in A_{1} \rightarrow b \in A_{1}$.

DEFINITION 1.3. Let $C$ be a class of regular cardinals. We shall define two generalized quantifiers $\left(Q_{C}^{\mathrm{cf}} x, y\right)$ and $\left(Q_{C}^{\mathrm{d}} \mathrm{c} x, y\right)$ :

(A) $M \vDash\left(Q_{C}^{\text {cf }} x, y\right) \varphi(x, y ; \bar{a}) \Longleftrightarrow$ the relation $x<y \equiv_{\operatorname{def}} \varphi(x, y ; \bar{a})$ linearly orders $A=\{b \in M: M \vDash(\exists x) \varphi(x, b ; \bar{a})\}$ and $\operatorname{cf}(A,<) \in C$.

(B) $M \vDash\left(Q_{C}^{\mathrm{dc}} x, y\right) \varphi(x, y ; \bar{a}) \Longleftrightarrow$ the relation $x<y \equiv_{\mathrm{def}} \varphi(x, y ; \bar{a})$ linearly orders $A=\{b \in M: M \vDash(\exists x) \varphi(x, b ; \bar{a})\}$ and there is a Dedekind cut $\left(A_{1}, A_{2}\right)$ of $(A, \succ)$ such that $\operatorname{cf}\left(A_{1}, \succ\right), \operatorname{cf}^{*}\left(A_{2}, \succ\right) \in C$. Clearly the syntax of $L\left(Q_{C}^{\mathrm{cf}}, Q_{C}^{\mathrm{dc}}\right)$, the logic obtained by adding the two generalized quantifiers to first-order logic, is not dependent on $C$.

DEFINITION 1.4. $L^{*}=L\left(Q_{\left\{\kappa_{0}, \kappa\right\}}^{\text {cf }}, Q_{\left\{\kappa_{0}, \kappa\right\}}^{d c}\right)$ where $\kappa$ is the first weakly compact cardinal. In the following we shall omit writing $\left\{\aleph_{0}, \kappa\right\}$.

LEMMA 1.2. $L^{*}$ is stronger than $L$ for countable models.

Proof. We must find a sentence $\psi \in L^{*}$ for which there is no $\psi^{\prime} \in L$ such that for every countable model $M, M \vDash \psi \Longleftrightarrow M \vDash \psi^{\prime}$. 
Let $\psi=[<$ is a linear order $] \wedge$ [every element has an immediate follower and an immediate predecessor] $\wedge \neg\left(Q^{\mathrm{dc}} x, y\right)(x<y)$.

Clearly a countable order satisfies $\psi$ iff it is isomorphic to the order of the integers. So clearly there is no sentence of $L$ equivalent to $\psi$ for countable models.

THEOREM 1.3. $L^{*}$ is compact.

REMARK. If we just wanted to prove $\lambda$-compactness for $\lambda<\kappa$, the proof would be somewhat easier.

In order to take care of the possibility that $|L| \geqslant \kappa$, we encode all the $m$-place relations by one relation with parameters and then we use saturativity. A similar trick was used by Chang [Ch 2] who attributes it to Vaught who attributes it [Va 1] to Chang.

We also use the technique of indiscernibles from Ehrenfeucht-Mostowski [EM]. Helling [He 1] used indiscernibles with weakly compact cardinals.

Proof of Theorem 1.3. Let $T$ be a theory in $L^{*}$ such that every finite subtheory $t \subseteq T$ has a model. We must show that $T$ has a model. Without loss of generality we may make the following assumptions.

Assumption 1. There is a singular cardinal $\lambda_{0}>|T|+\kappa$ such that every (finite) $t \subseteq T$ has a model of power $\lambda_{0}$. (There is clearly a singular $\lambda_{0}>_{k}+$ $|T|$ such that every $t \subseteq T$ has a model of power $<\lambda_{0}$. Now let $P$ be a new one-place predicate symbol, and replace every sentence of $T$ by its relativization to $P$ (i.e. replace $\left(Q^{\mathrm{cf}} x, y\right) \varphi(x, y, \bar{z})$ by $\left(Q^{\mathrm{cf}} x, y\right)(P(x) \wedge P(y) \wedge \varphi(x, y, \bar{z}))$ and replace $\left(Q^{\mathrm{dc}} x, y\right) \varphi(x, y, \bar{z})$ by $\left.\left(Q^{\mathrm{dc}} x, y\right)(P(x) \wedge P(y) \wedge \varphi(x, y, \bar{z}))\right)$. Let $T^{\prime}$ be the resulting theory. Clearly every $t \subseteq T^{\prime}$ has a model of power $\lambda_{0}$, and $T^{\prime}$ has a model iff $T$ has a model. Also $\left|T^{\prime}\right|=|T|$.

Assumption 2. Every $t \subseteq T$ has a model $M_{t}$ (of power $\lambda_{0}$ ) whose universe set is $\lambda_{0}=\left\{\alpha: \alpha<\lambda_{0}\right\},<$ (the order on the ordinals) is a relation of $M_{t}, R C^{M_{t}}=\left\{\mu: \mu<\lambda_{0}\right.$ is a regular cardinal $\}, \omega$ and $\kappa$ are individual constants, and there is a pairing function.

Assumption 3. There is $L_{a} \subseteq L, L_{a}$ countable, and the only symbols in $L-L_{a}$ are individual constants, and $\omega, \kappa$ are in $L_{a}$. We can assume that $L$ has no function symbols.

Let $\left\{R_{i}^{n}: i<\alpha_{n}, n<\omega\right\}$ be a list of all the predicate symbols in $L$, $R_{i}^{n}$ being $n$-place. Define languages $L_{0}^{\prime}, L_{1}^{\prime}$ as follows: $L_{1}^{\prime}=\{\omega, \kappa,<\} \cup$ $\left\{R^{n}: n<\omega, R^{n}\right.$ is an $(n+1)$-place predicate symbol $\}, L_{0}^{\prime}=L_{1}^{\prime} \cup\left\{c_{i}^{n}: i<\right.$ $\alpha_{n}, n<\omega, c_{i}^{n}$ individual constant symbol\}. If $\psi \in T$ define $\psi_{0}$ by replacing every occurrence of $R_{i}^{n}\left(x_{1}, \cdots, x_{n}\right)$ in $\psi$ by $R^{n}\left(x_{1}, \cdots, x_{n}, c_{i}^{n}\right)$. Let $T_{0}=\left\{\psi_{0}: \psi \in T\right\}, T_{0}$ is a theory in $L_{0}^{\prime *}$ and may be taken in place of $T$. 
Claim 1.4. For every language $L_{b}$ containing $<$ there is a language $L_{c}$ and a theory $T_{c}=T\left(L_{b}\right)$ in $L_{c}^{*}$ such that:

(1) $L_{b} \subseteq L_{c},\left|L_{b}\right|=\left|L_{c}\right|$.

(2) Every model $M_{b}$ for $L_{b}$ has a fixed expansion to a model $M_{c}$ for , $L_{c}$ which is a model of $T_{c}$.

(3) Every formula in $L_{c}^{*}$ is $T_{c}$-equivalent to an atomic formula; i.e. for all $\varphi(\bar{x}) \in L_{c}^{*}$ there is a predicate symbol $R_{\varphi}(\bar{x})$ such that $(\forall \bar{x})(\varphi(\bar{x}) \equiv$ $\left.R_{\varphi}(\bar{x})\right) \in T_{c}$.

(4) $T_{c}$ has Skolem functions; i.e., for all $\varphi(y, \bar{x}) \in L_{c}^{*}$ there is a function symbol $F_{\varphi} \in L_{c}^{*}$ such that

$$
(\forall \bar{x})\left[(\exists y) \varphi(y, \bar{x}) \equiv \varphi\left(F_{\varphi}(\bar{x}), \bar{x}\right)\right] \in T_{c} .
$$

(5) For every formula $\varphi(x, y, \bar{z}) \in L_{c}^{*}$ there are function symbols $F_{\varphi}^{i} \in$ $L_{c}$ (for $i=1, \cdots, 5$ ) such that: if $\left|M_{b}\right|=\lambda_{0}$ (the universe set of $M_{b}$ ), $<^{M_{b}}$ is the "natural" order, then for all sequences $\bar{a}$ from $M_{b}$ if $\varphi(x, y, \bar{a})$ linearly orders $A=\left\{y \in\left|M_{c}\right|: M_{c} \vDash(\exists x) \varphi(x, y, \bar{a})\right\} \neq \varnothing$ then (in $\left.M_{c}\right)$ :

(i) $F_{\varphi}^{1}(\bar{a})=\operatorname{cf}(A, \varphi(x, y, \bar{a}))$.

(ii) The sequence $\left\langle F_{\varphi}^{2}(y, \bar{a}): y\left\langle F_{\varphi}^{1}(\bar{a})\right\rangle\right.$ is an increasing unbounded sequence in $A$.

(iii) $A$ has a cut $\left(A_{1}, A_{2}\right)$ such that $\operatorname{cf}^{*}\left(A_{2}, \varphi(x, y, \bar{a})\right)=\mu$, $\operatorname{cf}\left(A_{1}, \varphi(x, y, \bar{a})\right)=\chi$ iff $F_{\varphi}^{3}(\mu, \chi, \bar{a})=0$ iff $F_{\varphi}^{3}(\mu, \chi, \bar{a}) \neq 1$.

(iv) If $F_{\varphi}^{3}(\mu, \chi, \bar{a})=0$ then $\left\langle F_{\varphi}^{4}(y, \mu, \chi, \bar{a}): y\langle\chi\rangle\right.$ is an increasing unbounded sequence in $A_{1}$.

(v) If $F_{\varphi}^{3}(\mu, \chi, \bar{a})=0$ then $\left\langle F_{\varphi}^{5}(y, \mu, \chi, \bar{a}): y\langle\mu\rangle\right.$ is a decreasing unbounded sequence in $A_{2}$ [where $A_{1}, A_{2}$ in (iv), (v) are from (iii)].

Proof. If in each stage we were to take $\varphi \in L_{b}^{*}$ (instead of $L_{c}^{*}$ ) the proof would be trivial. By repeating this process $\omega$ times we get the desired result.

Notation. Define languages $L_{n}$ and theories $T_{n}$ in $L_{n}^{*}$ as follows: $L_{0}=$ $L_{a} \cup\{P\}$ where $L_{a}$ is from Assumption 3 and $P$ is a new unary predicate symbol. If $L_{n}$ is defined let $L_{n}^{\prime}=L_{n} \cup\left\{P_{n}, P^{n}\right\}$ where $P_{n}, P^{n}$ are new unary predicate symbols. Now $L_{n+1}, T_{n+1}$ will be $L_{c}$ and $T\left(L_{b}\right)$ from Claim 1.4 where $L_{n}^{\prime}$ corresponds to $L_{b}$. Clearly $L_{n}$ are countable. Let $L_{\infty}=\bigcup L_{n}, T_{\infty}=\bigcup T_{n}$.

DEFinition 1.4. If $M$ is a model, $\Delta$ a set of formulas $\varphi(\bar{x})$ (i.e. a formula with a finite sequence of variables, including its free variables) in the language of $M, A \subseteq|M|$, then the sequence $\left\{b_{i}: i<\alpha\right\} \subseteq|M|$ is $\Delta$-indiscernible (or a sequence of $\Delta$-indiscernibles) over $A$ if $i \neq j \Rightarrow b_{i} \neq b_{j}$ and for all $\varphi\left(x_{0}, \cdots, x_{k-1}\right) \in \Delta, n \leqslant k$, permutation $\sigma$ of $\{0, \cdots, n-1\}$ and 
$a_{n}, \cdots, a_{k-1} \in A$ and $j(0)<\cdots<j(n-1)<\alpha, i(0)<\cdots<i(n-1)<\alpha$ the following holds:

$$
\begin{aligned}
M & \neq\left[b_{i(\sigma(0))}, \cdots, b_{i(\sigma(n-1))}, a_{n}, \cdots, a_{k-1}\right] \\
& \Longleftrightarrow M \vDash\left[b_{j(\sigma(0))}, \cdots, b_{j(\sigma(n-1))}, a_{n}, \cdots, a_{k-1}\right] .
\end{aligned}
$$

Claim 1.5. 1. If $A, \Delta, M$ are as in Definition 1.4, $A$ and $\Delta$ are finite, and $B \subseteq|M|$ is infinite, then there are $b_{i} \in B$ such that $\left\{b_{i}: i<\omega\right\}$ is $\Delta$ indiscernible over $A$.

2. If $A, \Delta, M$ are as in Definition 1.4, $\Delta$ is finite, $B \subseteq|M|,|A|<\kappa \leqslant$ $|B|$, then there are $b_{i} \in B$ such that $\left\{b_{i}: i<\kappa\right\}$ is $\Delta$-indiscernible over $A$ ( $\kappa$ is the weakly compact cardinal chosen at the beginning).

Proof. 1. This is a result of the infinite Ramsey theorem. EhrenfeuchtMostowski [EM] used this to obtain essentially (1).

(2) It is known that $\kappa$ is weakly compact iff $\kappa \rightarrow(\kappa)_{\mu}^{m}$ for all $\mu<\kappa$ (see [Si 1]). From here the result is immediate.

Let $\left\{c_{\alpha}: \alpha<\alpha_{T}\right\}$ be all the individual constants in $L-L_{a}$ (see Assumption 3). Let $S=\left\{(t, n, B): t \subseteq T, n<\omega, B \subseteq\left\{c_{\alpha}: \alpha<\alpha_{T}\right\}, t\right.$ and $B$ finite $\}$. Denote elements of $S$ by $s$ or $s_{i}=\left(t_{i}, n_{i}, B_{i}\right)$ and $s_{1} \leqslant s_{2}$ will mean $t_{1} \subseteq$ $t_{2}, n_{1} \leqslant n_{2}, B_{1} \subseteq B_{2}$. Now we define the $L_{n}$-model $M(s), s=(t, n, B)$. For $t, B$ fixed, denote $M(s)$ by $M^{n}$. Define $M^{n}$ by induction on $n$ such that $M^{n+1}$ expands $M^{n}, M^{n}$ is an $L_{n}$-model, $P_{n}\left(M^{n+1}\right) \subseteq \omega, P^{n}\left(M^{n+1}\right) \subseteq \kappa$, $\left|P_{n}\left(M^{n+1}\right)\right|=\aleph_{0},\left|P^{n}\left(M^{n+1}\right)\right|=\kappa$. For $n=0$ take $M^{0}$ to be the expansion of $M_{t}$ by adding the predicate $P\left(M^{0}\right)=B$. Let $\left\{\varphi_{i}\left(\bar{x}^{i}\right): i<\omega\right\}$ be a list of the formulas of $L_{\infty}$, such that the number of variables in $\bar{x}^{i}$ is $\leqslant i$, and let $\Delta_{n}=\left\{\varphi_{i}: i \leqslant n\right\} \cap L_{n}$. If $M^{n}$ is defined we define $M^{n+1}$ as follows: Let $A^{1} \subseteq P^{n-1}\left(M^{n}\right)$ (or $A^{1} \subseteq\{a: a<\kappa\}$ if $n=0$ ) be a $\Delta_{n}$-indiscernible sequence over $B \cup\{a: a<\omega\}$ and let $A^{2} \subseteq P_{n-1}\left(M^{n}\right)$ (or $A^{2} \subseteq\{a: a<\omega\}$ if $n=0)$ be a $\Delta_{n}$-indiscernible sequence over $B \cup\left\{a^{1}, \cdots, a^{n}\right\}$, where $a^{1}$, $\cdots, a^{n}$ are the first $n$ elements of $A^{1}$. (In fact $A^{1}, A^{2}$ are sets, but we look on them as sequences by the ordering <.) As for each $\varphi(\bar{x}) \in \Delta_{n}$ the number of variables in $\bar{x}$ is $\leqslant n, A^{2}$ is $\Delta_{n}$-indiscernible over $B \cup A^{1}$. Expand $M^{n}$ by interpreting $P^{n}$ as $A^{1}$ and $P_{n}$ as $A^{2}$, and then expand the result to an $L_{n+1}$-model by Claim 1.4, so it will be a model of $T_{n}$ (mentioned in the notation after Claim 1.4). This will be $M^{n+1}$. Let $L_{U}$ be the language obtained from $L_{\infty}$ by adding the individual constants $\left\{c_{\alpha}: \alpha<\alpha_{T}\right\}$ (from $L-$ $L_{a}$ ) and new constants $y^{i}, y_{i}$ for $i<\kappa$. Now we define a first-order theory $T_{U}$ in $L_{U}$. Let $\psi\left(x_{1}, \cdots, x_{l} ; x^{1}, \cdots, x^{m} ; z_{1}, \cdots, z_{k}\right)$ be a formula in $L_{\infty}$ and let $j(1)<\cdots<j(m)<\kappa, i(1)<\cdots<i(l)<\kappa$. Then 


$$
\psi\left(y_{i(1)}, \cdots, y_{i(l)} ; y^{j(1)}, \cdots, y^{j(m)} ; c_{\alpha(1)}, \cdots, c_{\alpha(k)}\right) \in T_{U}
$$

iff there is $s_{1} \in S$ such that, for all $s \geqslant s_{1}, s=(t, n, B)$, and for all $a_{1}<$ $\cdots<a_{l} \in P_{n}(M(s)), b_{1}<\cdots<b_{m} \in P^{n}(M(s))$, it is the case that

$$
M(s) \vDash \psi\left[a_{1}, \cdots, a_{l} ; b_{1}, \cdots, b_{m} ; c_{\alpha(1)}, \cdots, c_{\alpha(k)}\right] .
$$

Clearly $T_{U}$ is consistent. Let $M \vDash T_{U}$ be $\kappa^{+}$-saturated (see Morley and Vaught [MV] or e.g. Chang and Keisler [CK]). Let $N$ be the submodel of $M$ whose universe set is the closure of $P^{M}$ under the functions of $M$ (and so in particular all the individual constants are in $N$ ). Let $D$ be a nonprincipal ultrafilter on $\omega$, and let $N^{*}=N^{\omega} / D$. We shall show that $N^{*} \vDash T$, and thus complete the proof of the theorem. We use the fact that $N^{*}$ is $\aleph_{1}$-saturated (see e.g. [CK]).

Because of Claim 1.4(3) it is sufficient to show:

(I) If $R_{1}(x, y, \bar{z})$ is an atomic formula in $L_{\infty}$ and $(\forall \bar{z})\left[\left(Q^{\mathrm{cf}} x, y\right)\right.$ $\left.R_{1}(x, y, \bar{z}) \equiv R_{2}(\bar{z})\right] \in T_{\infty}$, then for all $\bar{a} \in N^{*}$

$$
N^{*} \vDash\left(Q^{\mathrm{cf}} x, y\right) R_{1}(x, y, \bar{a}) \Longleftrightarrow N^{*} \vDash R_{2}[\bar{a}] .
$$

(II) If $R_{1}(x, y, \bar{z})$ is an atomic formula in $L_{\infty}$ and $(\forall z)\left[\left(Q^{\mathrm{dc}} x, y\right)\right.$ $\left.R_{1}(x, y, \bar{z}) \equiv R_{2}(\bar{z})\right] \in T_{\infty}$, then for all $\bar{a} \in N^{*}$

$$
N^{*} \vDash\left(Q^{\mathrm{dc}} x, y\right) R_{1}(x, y, \bar{a}) \Longleftrightarrow N^{*} \vDash R_{2}[\bar{a}] .
$$

Proof of(I). Clearly the sets $\left\{a \in N^{*}: a<\omega\left(N^{*}\right)\right\},\left\{a \in N^{*}: a<\kappa\left(N^{*}\right)\right\}$ are linearly ordered by $<$, and both have cofinality $\kappa$. So by the assumptions and Claim 1.4(5), $N^{*} \vDash R_{2}(\bar{a}) \Rightarrow N^{*} \vDash\left(Q^{\mathrm{cf}} x, y\right) R_{1}(x, y, \bar{a})$.

Now assume $N^{*} \vDash \neg R_{2}[\bar{a}]$ but $N^{*} \vDash\left(Q^{\mathrm{cf}} x, y\right) R_{1}(x, y, \bar{a})$. We shall produce a contradiction. Hence $R_{1}(x, y, \bar{a})$ linearly orders $A=\left\{b: N^{*} \vDash\right.$ $\left.(\exists x) R_{1}(x, b, \bar{a})\right\} \neq \varnothing$, and $A$ has no last element. Since $N^{*}$ is $\aleph_{1}$-saturated, cf $A>\aleph_{0}$ and so by $N^{*} \vDash\left(Q^{\text {cf }} x, y\right) R_{1}(x, y, \bar{a})$ we have that $\operatorname{cf} A=\kappa$. By the assumptions and Claim 1.4(5)(ii) we may assume that $R_{1}(x, y, \bar{a})=x<$ $y \wedge y<a$ (a is one element in place of the sequence $\bar{a}), N^{*} \vDash R C[a]$, and so $A=\left\{b: N^{*} \vDash b<a\right\}$. Let $\left\{a_{i}\right\}_{i<\kappa}$ be an increasing unbounded sequence in $A, a_{\kappa}=a$, and suppose that $a_{i}=\left\langle\cdots, a_{i}^{n}, \cdots\right\rangle_{n<\omega} / D$ where $a_{i}^{n} \in N$ (since $\left.N^{*}=N^{\omega} / D\right)$.

Now for all $\alpha<\beta<\kappa$ define $f(\alpha, \beta)=\left\{n<\omega: a_{\alpha}^{n}<a_{\beta}^{n}<a_{\kappa}^{n}, R C\left[a_{\kappa}^{n}\right]\right.$, $\left.a_{\kappa}^{n} \neq \omega, \kappa\right\}$. Since $N^{*} \vDash\left(a_{\alpha}<a_{\beta}<a_{\kappa} \wedge R C\left[a_{\kappa}\right] \wedge a_{\kappa} \neq \omega \wedge a_{\kappa} \neq \kappa\right)$ we have by Łos' theorem that $f(\alpha, \beta) \in D$. $\kappa$, being weakly compact, satisfies $\kappa \rightarrow(\kappa)_{2}^{2} \aleph_{0}$ and so without loss of generality $f(\alpha, \beta)=f(0,1)$. If, for all $n \in f(0,1)$, there exists $b^{n}$ such that $a_{\alpha}^{n}<b^{n}<a_{\kappa}^{n}$ for all $\alpha \in \kappa$, then $b=\left\langle\cdots, b^{n}, \cdots\right\rangle \mid D \in N^{*}$ and $a_{\alpha}<b<a$ for all $\alpha<\kappa$, a contradiction. 
So there is $n \in f(0,1)$ for which $\left\{a_{\alpha}^{n}: \alpha<k\right\}$ is an (increasing) unbounded sequence in $\left\{b \in N: b<a^{n}\right\}$ and $N \vDash R C\left[a^{n}\right] \wedge a^{n} \neq \omega \wedge a^{n} \neq \kappa$. From now on denote $a=a^{n}, a_{\alpha}=a_{\alpha}^{n}$. Let $a_{\alpha}=\tau_{\alpha}\left(\cdots, y^{j(\alpha, m)}, \cdots ; \cdots, y_{i(\alpha, l)}\right.$, $\left.\cdots ; \bar{b}_{\alpha}\right)_{l<l(\alpha), m<m(\alpha)}$, where $\tau_{\alpha}$ is a term, in $L_{\infty}, j(\alpha, m)$ is an increasing sequence in $m, i(\alpha, l)$ is an increasing sequence in $l$, and $\bar{b}_{\alpha}$ is a sequence from $P^{N}$. Since we may replace $\left\{a_{\alpha}: \alpha<\kappa\right\}$ by any subset of the same power, we may assume that $m(\alpha)=m_{0} . l(\alpha)=l_{0}$, and $\tau_{\alpha}=\tau$ for all $\alpha<k$.

Since $N \vDash R C[a] \wedge a>\omega$ and in every $M(s)$ the interpretation of $P$ is a finite set, and $\{b: b<\omega\}$ is a countable set, there is a function symbol $F$ in $L_{\infty}$ such that

$$
\begin{aligned}
& F\left(x^{0}, \cdots,\right.\left.x^{m_{0}-1}, x\right) \\
&=\sup \left\{\tau\left(x^{0}, \cdots, x^{m_{0}-1} ; z_{0}, \cdots, z_{l_{0}-1}, v_{1}, \cdots\right)<x:\right. \\
& \\
&\left.z_{0}, \cdots,<\omega, v_{1}, \cdots, \in P\right\} .
\end{aligned}
$$

Clearly $\tau\left(\cdots, y^{j(\alpha, m)}, \cdots ; \cdots, y_{i(\alpha, l)}, \cdots ; \bar{b}_{\alpha}\right)<F\left(\cdots, y^{j(\alpha, m)}, \cdots, a\right)$ $<a$, and thus without loss of generality $a_{\alpha}=F\left(\cdots, y^{j(\alpha, m)}, \cdots, a\right)$. If $N \vDash a<\kappa$ then $N$ satisfies the sentence "saying:" there is a regular cardinal $a<\kappa$ such that $X_{\kappa}$ is an unbounded subset of $\{c: c<a\}$, but $X_{b}$ is a bounded subset of $\{c: c<a\}$ for any $b<k$; where $X_{b}=\{F(\cdots, x, \cdots, a)$ $<a: x<b\}$. Hence, for some s, $M(s)$ satisfies it, contradicting the fact that cf $\kappa=\kappa$. If $N \vDash a>k$, as we get $F$ we can get $F^{\prime}$ such that $a_{\alpha}<F^{\prime}(a)<$ $a$ for every $\alpha$, a contradiction.

Proof of (II). As in the proof of (I) it is clear by Claim 1.4 that $N^{*} \vDash$ $R_{2}[\bar{a}] \Rightarrow N^{*} \vDash\left(Q^{\mathrm{dc}} x, y\right) R_{1}(x, y, \bar{a})$.

Now assuming $N^{*} \vDash\left(Q^{\mathrm{dc}} x, y\right) R_{1}(x, y, \bar{a}) \wedge \neg R_{2}(\bar{a})$ we shall arrive at a contradiction. We can restrict ourselves to the case where $x<y \equiv_{\mathrm{def}} R_{1}(x, y ; \bar{a})$ linearly orders $A=\left\{b \in N^{*}:(\exists x) R_{1}(x, b, \bar{a})\right\} \neq \varnothing, A$ has no last element. Since there are pairing functions we may replace $\bar{a}$ by $a$. By hypothesis $A$ has a Dedekind cut $\left(A_{1}, A_{2}\right)$ such that $\operatorname{cf} A_{1}, \mathrm{cf}^{*} A_{2} \in\{\omega, \kappa\}$.

Case 1. $\operatorname{cf} A_{1}=\mathrm{cf}^{*} A_{2}=\omega$ : This contradicts the $\aleph_{1}$-saturation of $N^{*}$.

Case 2. cf $A_{1}=\omega, \mathrm{cf}^{*} A_{2}=\kappa$ : Let $\left\{b_{m}\right\}_{m<\omega}$ be an increasing unbounded sequence in $A_{1}$, and let $\left\{a_{\alpha}\right\}_{\alpha<\kappa}$ be a decreasing unbounded sequence in $A_{2}$, where $b_{m}=\left\langle\cdots, b_{m}^{n}, \cdots\right\rangle_{n<\omega} / D, a_{\alpha}=\left\langle\cdots, a_{\alpha}^{n}, \cdots\right\rangle_{n<\omega} / D$.

For all $\alpha<\kappa$ define $f_{1}(\alpha)=\left\langle\left\{n<\omega: b_{m}^{n}<a_{\alpha}^{n}\right\}: m<\omega\right\rangle$. Since the range of $f_{1}$ is a set of power $\leqslant 2^{\aleph_{0}}$ we can assume that $f_{1}$ is constant. Let $T_{m}=\left\{n<\omega: b_{m}^{n}<a_{\alpha}^{n}\right\}$; clearly $T_{m} \in D$. Let $R$ be a new one-place predicate symbol, $R^{n}=\left\{b_{m}^{n}: n \in T_{m}\right\}$, and $\left(N^{*}, R\right)=\Pi_{n<\omega}\left(N, R^{n}\right) / D$. Clearly $\left\{b_{m}: m<\omega\right\} \subseteq R \cap A$ and $\left\langle R \cap A,<^{*}\right\rangle$ is an $\aleph_{1}$-saturated model of the 
theory of order, and so it contains an upper bound to the $b_{m}$ 's, and also $b<^{*} a_{\alpha}$ for all $b \in R \cap A, \alpha<\kappa$. This is a contradiction.

Case 3. cf $A_{1}=\kappa, \mathrm{cf}^{*} A_{2}=\omega$ : The proof is similar to the proof of Case 2 .

Case 4. cf $A_{1}=\mathrm{cf}^{*} A_{2}=\kappa$ : Let $\left\{a_{\alpha}\right\}_{\alpha<\kappa}\left(\left\{b_{\alpha}\right\}_{\alpha<k}\right)$ be an increasing (decreasing) unbounded sequence in $A_{1}\left(A_{2}\right)$, where $a_{\alpha}=\left\langle\cdots, a_{\alpha}^{n}, \cdots\right\rangle_{n \in \omega} / D$, $b_{\alpha}=\left\langle\cdots, b_{\alpha}^{n}, \cdots\right\rangle_{n \in \omega} / D$.

As in (I) we can assume that for all $\alpha<\beta<\kappa$ the following sets are not dependent on the particular $\alpha$ or $\beta$ :

$$
J_{1}=\left\{n<\omega: a_{\alpha}^{n}<a_{\beta}^{n}\right\}, \quad J_{2}=\left\{n<\omega: a_{\alpha}^{n}<b_{\beta}^{n}\right\}, \quad J_{3}=\left\{n<\omega: b_{\beta}^{n}<b_{\alpha}^{n}\right\} .
$$

Also $J_{i} \in D$, and $J_{0}=\left\{n<\omega: N \vDash \neg R_{2}\left[a^{n}\right]\right\} \in D$, where $a=\left\langle\cdots, a^{n}\right.$, $\cdots$. Thus as in (I), for some $n \in \bigcap_{i}, R_{1}\left(x, y, a^{n}\right)$ linearly orders

$$
A=\left\{y \in N:(\exists x) R_{1}\left(x, y, a^{n}\right)\right\} \supseteq\left\{a_{\alpha}^{n}, b_{\alpha}^{n}: \alpha<\kappa\right\}
$$

and, for no $c \in A, a_{\alpha}^{n}<c<b_{\alpha}^{n}$. So by renaming,

(*) There is $a \in N, N \vDash \neg R_{2}[a], A=\left\{b \in N: N \vDash(\exists x) R_{1}(x, b, a)\right\}$ is linearly ordered by $x<^{*} y=R_{1}(x, y, a)$, and $A$ has a cut $\left(A_{1}, A_{2}\right)$ with $\left\{a_{\alpha}\right\}_{\alpha<\kappa}\left(\left\{b_{\alpha}\right\}_{\alpha<\kappa}\right)$ an increasing (decreasing) unbounded sequence in $A_{1}$ $\left(A_{2}\right)$. Let

$$
a_{\alpha}=\tau_{\alpha}\left(\cdots, y^{j(\alpha, l)}, \cdots ; \cdots, y_{i(\alpha, m)}, \cdots ; \bar{d}_{\alpha}\right)_{l<l(\alpha), m<m(\alpha)},
$$

and $j(\alpha, l)$ and $i(\alpha, m)$ increase with $l, m$ respectively, $a=$ $\tau^{*}\left(\cdots, y^{\xi(l)}, \cdots ; \cdots, y_{\zeta(m)}, \cdots ; \bar{d}\right)$ : where $\bar{d}, \bar{d}_{\alpha}$ are sequences from $P^{M}=P^{N}$.

Since $k$ is weakly compact we can assume the following:

(1) $\tau_{\alpha}=\tau_{0}, l(\alpha)=l(0), m(\alpha)=m(0)$.

(2) For every formula $\varphi\left(\bar{x}^{1}, \bar{x}^{2}, \bar{x}^{3}\right) \in L_{\infty}$ the truth value of $\varphi\left(\bar{d}_{\alpha}, \bar{d}_{\beta}, \bar{d}\right)$ is the same for all $\alpha<\beta<\kappa$.

(3) There is $l_{1}<l(0)$ such that for every $\alpha<\beta<\kappa$

$$
\begin{aligned}
y^{j(\alpha, 0)} & =y^{j(\beta, 0)}<y^{j(\alpha, 1)}=y^{j(\beta, 1)}<\cdots<y^{j\left(\alpha, l_{1}-1\right)}=y^{j\left(\beta, l_{1}-1\right)} \\
& <y^{j\left(\alpha, l_{1}\right)}<y^{j\left(\alpha, l_{1}+1\right)}<\cdots<y^{j(\alpha, l(0)-1)}<y^{j\left(\beta, l_{1}\right)} \\
& <\cdots<y^{j(\beta, l(0)-1)}
\end{aligned}
$$

and $y^{\xi(l)}<y^{j\left(\alpha, l_{1}\right)}$ fo- any $l$. Denote for $l<l_{1} y^{j(l)}=y^{j(\alpha, l)}$,

$$
\begin{aligned}
& \bar{y}^{*}=\left\langle y^{j(0)}, \cdots, y^{j\left(l_{1}-1\right)}, \cdots, y^{\xi(l)}, \cdots\right\rangle, \\
& \bar{y}^{\alpha}=\left\langle y^{j\left(\alpha, l_{1}\right)}, \cdots, y^{j(\alpha, l(0)-1)}\right\rangle .
\end{aligned}
$$


(4) Similar to (3) for the $y_{i(\alpha, m)}$, we get $\bar{y}_{\alpha}$ and $\bar{y}_{*}$. Thus $a_{\alpha}=$ $\tau_{0}\left(\bar{y}^{*}, \bar{y}^{\alpha}, \bar{y}_{*}, \bar{y}_{\alpha}, \bar{d}_{\alpha}\right), a=\tau^{*}\left(\bar{y}^{*}, \bar{y}_{*}, \bar{d}\right)$. By treating the $b_{\alpha}$ similarly aniu making some change in $\bar{y}^{*}, \bar{y}_{\alpha}, \bar{d}_{\alpha}$ we may assume

(5) $b_{\alpha}=\tau^{0}\left(\bar{y}^{*}, \alpha \bar{y}, \bar{y}_{*}, \alpha \bar{y}, \bar{d}^{\alpha}\right)$, and if $\alpha<\beta$ then every element of $\alpha \dot{\bar{y}}$ comes before every element of $\beta \bar{y}$ (in the sequence $\left\{y^{i}: i<\kappa\right\}$ ), and after every element of $\bar{y}^{*}$. Similarly for ${ }_{\alpha} \bar{y}$. (Of course $\bar{d}^{\alpha}$ is a sequence from $P^{M} ; \bar{y}^{*},{ }^{\alpha} \bar{y}$ from $\left\{y^{i}: i<\kappa\right\}$ and $\bar{y}_{*}, \bar{y}_{\bar{y}}$ from $\left\{y_{i}: i<\kappa\right\}$.)

(6) As a strengthening of (2), for all $\varphi\left(\bar{x}^{1}, \bar{x}^{2}, \bar{x}^{3}\right) \in L_{\infty}$ and all $\alpha, \beta$ the truth values of $\varphi\left(\bar{d}^{\alpha}, \bar{d}^{\beta}, \bar{d}\right), \varphi\left(\bar{d}_{\alpha}, \bar{d}^{\beta}, \bar{d}\right)$, and $\varphi\left(\bar{d}_{\alpha}, \bar{d}_{\beta}, \bar{d}\right)$ are dependent only on the order between $\alpha$ and $\beta$.

Notation. $a_{\alpha, \beta, \gamma}=\tau_{0}\left(\bar{y}^{*}, \bar{y}^{\alpha}, \bar{y}_{*}, \bar{y}_{\beta}, \bar{d}_{\gamma}\right), b_{\alpha, \beta, \gamma}=\tau^{0}\left(\bar{y}^{*}, \alpha \bar{y}, \bar{y}_{*, \beta} \bar{y}, \bar{d}^{\gamma}\right)$.

Notice that by the indiscernibility of the $y$ 's and (6), $a_{\alpha, \beta, \gamma}, b_{\alpha, \beta, \gamma} \in A$ and the order between $a_{\alpha, \beta, \gamma}$ and $a_{\alpha(1), \beta(1), \gamma(1)}$ depends only on the order between $\alpha$ and $\alpha(1)$, the order between $\beta$ and $\beta(1)$, and the order between $\gamma$ and $\gamma(1)$; and similarly for the $b_{\alpha, \beta, \gamma}$.

Now for every $\alpha, \beta, \gamma, \delta<\kappa$ choose $\epsilon, \alpha, \beta, \gamma, \delta<\epsilon<\kappa$. So $a_{\alpha}<$ $b_{\epsilon} \Rightarrow a_{\alpha, \alpha, \alpha}<b_{\epsilon} \Rightarrow a_{\alpha, \beta, \gamma}<b_{\epsilon} \Rightarrow a_{\alpha, \beta, \gamma}<b_{\delta}$, and hence every $a_{\alpha, \beta, \gamma} \in A_{1}$. Similarly $b_{\alpha, \beta, \gamma} \in A_{2}$.

If $a_{0,0,1} \leqslant a_{1,1,0}$ then $\alpha<\alpha(1), \beta>\beta(1)$ imply $a_{\alpha, \alpha, \beta} \leqslant a_{\alpha(1), \alpha(1), \beta(1)}$. So for all $\alpha>0, a_{\alpha, \alpha, \alpha} \leqslant a_{\alpha+1, \alpha+1,0}$, and so $\left\{a_{\alpha, \alpha, 0}: \alpha<k\right\}$ is an unbounded subset of $A_{1}$. Similarly, if $a_{0,0,1} \leqslant a_{1,1,0}$ and $a_{1,2,0} \leqslant a_{2,1,0}$ then $\left\{a_{\alpha, 1,0}: \alpha<\kappa\right\}$ is unbounded in $A_{1}$, if $a_{0,0,1} \leqslant a_{1,1,0}$ and $a_{1,2,0}>a_{2,1,0}$ then $\left\{a_{1, \alpha, 0}: \alpha<\kappa\right\}$ is unbounded in $A_{1}$, and if $a_{0,0,1}>a_{1,1,0}$ then $\left\{a_{0,0, \alpha}: \alpha<\kappa\right\}$ is unbounded in $A_{1}$. A parallel claim is true for the $b$ 's. So we may change $\tau_{0}$ and $\tau^{0}$ such that $a_{\alpha, \beta, \gamma}$ and $b_{\alpha, \beta, \gamma}$ will each be dependent only on one index. (If $a_{\alpha, \beta, \gamma}$ is not dependent on $\alpha$, then $\bar{y}^{\alpha}$ is empty; if not dependent on $\beta, \bar{y}_{\beta}$ is empty, and if not dependent on $\gamma, \bar{d}_{\gamma}$ is constant.) There are, in all, nine possibilities.

We shall now show that there cannot be dependence on $\gamma$ alone. Assume without loss of generality that $a_{\alpha}=\tau_{0}\left(\bar{y} ; \bar{d}_{\gamma}\right)$ where $\bar{y}$ is the concatenation of all sequences from $\left\{\bar{y}_{i}, \bar{y}^{i}: i<\kappa\right\}$ which are not dependent on $\gamma$. Consider the following type in the variables $x_{i}, i<l=l\left(\bar{d}_{\underline{\gamma}}\right)$ : (let $\bar{x}=\left\langle x_{1}, \cdots, x_{l}\right\rangle$ : $\left\{P\left(x_{i}\right): i<l\right\} \cup\left\{(\exists x) R_{1}\left(x, \tau_{0}(\bar{y}, \bar{x}), a\right)\right\} \cup\left\{\tau_{0}(\bar{y}, \bar{x})<b_{\alpha}: \alpha<\kappa\right\} \cup\left\{a_{\alpha}<\right.$ $\left.\left.\tau_{0}(\bar{y}, \bar{x}): \alpha<\kappa\right\}\right)$.

This type, containing parameters from $N$, is finitely satisfiable in $N$ and thus in $M$ since $N$ is an elementary submodel of $M$. Thus it is satisfiable by $\bar{c}=\left\langle c_{1}, \cdots, c_{l}\right\rangle$ in $M$, since $M$ is $\kappa^{+}$-saturated. But $c_{i} \in N$ since $c_{i} \in P^{M}$ and thus the type is satisfiable in $N$. This contradicts the definition of the $a_{\alpha}, b_{\alpha}$.

We are left with four cases. Without loss of generality we shall deal only 
with the case $a_{\alpha}=\tau_{0}\left(\bar{y}^{*}, \bar{y}^{\alpha}, \bar{y}_{*}, \bar{d}\right), b_{\alpha}=\tau^{0}\left(\bar{y}^{*}, \bar{y}_{*}, \bar{y}_{,} \bar{d}\right)$. Without loss of generality all the above sequences are of equal length, and it will be recalled that the sequences of the $y$ 's here are increasing sequences, $\bar{y}^{*}<\bar{y}^{\alpha}, \bar{y}_{*}<_{\alpha} \bar{y}$ (i.e., every element in the left sequence is smaller than every element in the matching right sequence).

For every sentence $\psi$ which $N$ satisfies and $s_{1} \in S$ there is $s \geqslant s_{1}$ such that $M(s)$ satisfies $\psi$. Hence there are $s \in S$, and a sequence $\vec{d} \in$ $P[M(s)]$ where $s=(t, n, B)$ such that $n>1000 l\left(y^{*}\right)$ and $n$ is big enough so that all the formulas we shall need are in $\Delta_{n-3}$ and (remembering the indiscernibility in the definition of $\left.P^{n-2}[M(s)], P_{n-2}[M(s)]\right)$.

$\left({ }^{* *}\right)$ If $\bar{c}^{*}<\bar{c}^{1}<\bar{c}^{2}$ are increasing sequences from $P^{n-2}[M(s)]$ and $\bar{c}_{*}<{ }_{1} \bar{c}<{ }_{2} \bar{c}$ are increasing sequences from $P_{n-2}[M(s)]$, and $l\left(\bar{c}^{*}\right)=l\left(\bar{y}^{*}\right)$, $l\left(\bar{c}^{2}\right)=l\left(\bar{c}^{1}\right)=l\left(\bar{y}^{1}\right), l\left(\bar{c}_{*}\right)=l\left(\bar{y}_{*}\right), l\left({ }_{1} \bar{c}\right)=l\left({ }_{2} \bar{c}\right)=l\left({ }_{1} \bar{y}\right)$ then

(A) $M(s) \vDash \neg R_{2}\left[\tau\left(\bar{c}^{*}, \bar{c}_{*}, \bar{d}^{\prime}\right)\right], R_{1}\left(x, y, \tau\left(\bar{c}^{*}, \bar{c}_{*}, \bar{d}^{\prime}\right)\right)$ is a linear order <* (nonempty) without a last element on a set $A_{s}$.

(B) In $M(s)$ the following holds:

$$
\begin{aligned}
\tau_{0}\left(\bar{c}^{*}, \bar{c}, \bar{c}_{*}, \bar{d}^{\prime}\right) & <\tau_{0}\left(\bar{c}^{*}, \bar{c}^{2}, \bar{c}_{*}, \bar{d}^{\prime}\right)<^{*} \tau^{0}\left(\bar{c}^{*}, \bar{c}_{*},{ }_{2} \bar{c}, \bar{d}^{\prime}\right) \\
& <* \tau^{0}\left(\bar{c}^{*}, \bar{c},{ }_{1} \bar{c}, \bar{d}^{\prime}\right) \in A_{s} .
\end{aligned}
$$

Define $A_{s}^{1}=\left\{b \in A_{s}:\right.$ there is $\bar{c}^{0}>\bar{c}^{*}$ such that $\left.b<^{*} \tau_{0}\left(\bar{c}^{*}, \bar{c}^{0}, \bar{c}_{*}, \bar{d}^{\prime}\right)\right\}$ and $A_{s}^{2}=\left\{b \in A_{s}:\right.$ there is $\bar{c}_{0}>\bar{c}_{*}$ such that $\left.\tau^{0}\left(\bar{c}^{*}, \bar{c}_{*}, \bar{c}_{0}, \bar{d}^{\prime}\right)\right\}<^{*} b$. Clearly $A_{s}^{1} \cap A_{s}^{2}=\varnothing$, cf $A_{s}^{1}=\kappa$, cf $A_{s}^{2}=\omega$, but from $M(s) \vDash \neg R_{2}\left[\tau\left(\bar{c}^{*}, \bar{c}_{*}, \bar{d}^{\prime}\right)\right]$ and by the definition of $R_{2}$ it follows that $\left.M(s) \vDash\right\urcorner\left(Q^{\mathrm{dc}} x, y\right) R_{1}\left[x, y, \tau\left(\bar{c}^{*}, \bar{c}, \bar{d}^{\prime}\right)\right]$. Thus there is $b \in A_{s}, A_{s}^{1}<b<A_{s}^{2}$. But $A_{s}, A_{s}^{1}, A_{s}^{2}$ are definable by the formulas $\varphi\left(x, \bar{c}^{*}, \bar{c}, \bar{d}^{\prime}\right), \varphi^{1}\left(x, \bar{c}^{*}, \bar{c}_{*}, \bar{d}^{\prime}\right), \varphi^{2}\left(x, \bar{c}^{*}, \bar{c}_{*}, \bar{d}^{\prime}\right)$, where $\varphi, \varphi^{1}, \varphi^{2} \in L_{n}$.

Now by 1.4 there is a function symbol $F$ in $L_{n+1}$ such that for all $s_{1}$ such that $n_{1}>n$ the following sentence holds in $M\left(s_{1}\right)$ (abusing our notation the free variables are $\left.\bar{y}_{*}, \bar{y}^{*}, \bar{z}\right)$ :

If $\neg R_{2}\left(\tau\left(\bar{y}^{*}, \bar{y}_{*}, \bar{z}\right)\right)$; and $R_{1}\left(x, y, \tau\left(\bar{y}^{*}, \bar{y}_{*}, \bar{z}\right)\right)$ defines a linear order on $A=\left\{v:(\exists x) R_{1}(x, v, \bar{z})\right\} ; \bar{y}_{*}\left(\bar{y}^{*}\right)$ is a sequence of elements $<\omega(<\kappa)$; and for all $\bar{y}^{*}<\bar{y}^{1}<\bar{y}^{2}$ such that the elements of $\bar{y}^{1}, \bar{y}^{2}$ are in $P^{n}$, and for all $\bar{y}_{*}<\bar{y}_{1}<\bar{y}_{2}$ such that the elements of $\bar{y}_{1}, \bar{y}_{2}$, are in $P_{n}$, it is true that

$$
\begin{aligned}
\tau_{0}\left(\bar{y}^{*}, \bar{y}^{1}, \bar{y}_{*}, \bar{z}\right) & <\tau_{0}\left(\bar{y}^{*}, \bar{y}^{2}, \bar{y}_{*}, \bar{z}\right) \\
& <^{*} \tau^{0}\left(\bar{y}_{1}, \bar{y}_{*}, \bar{y}_{2}, \bar{z}\right)<^{*} \tau^{0}\left(\bar{y}^{*}, \bar{y}_{*}, \bar{y}_{1}, \bar{z}\right) \in A
\end{aligned}
$$

where $x<^{*} y \equiv R_{1}\left(x, y, \tau\left(\bar{y}^{*}, \bar{y}_{*}, \bar{z}\right)\right)$, then $F\left(\bar{y}^{*}, \bar{y}_{*}, \bar{z}\right) \in A$ and for all $\bar{y}_{1}, \bar{y}^{1}$ as above 


$$
\tau_{0}\left(\bar{y}^{*}, \bar{y}^{1}, \bar{y}_{*}, z\right)<^{*} F\left(\bar{y}^{*}, \bar{y}_{*}, \bar{z}\right)<\tau^{0}\left(\bar{y}^{*}, \bar{y}_{*}, \bar{y}_{2}, \bar{z}\right) \text {. }
$$

Thus $M$, and $N$, satisfy the above sentence (because of the suitable indiscernibility of $\left.P^{n}, P_{n}\right)$. Thus $F\left(\bar{y}^{*}, \bar{y}_{*}, \bar{d}\right) \in A, a_{\alpha}<F\left(\bar{y}^{*}, \bar{y}_{*}, \bar{d}\right)<b_{\alpha}$, a contradiction. This concludes the proof of Theorem 1.3 and of Theorem 1.1.

2. Discussion. More on $L^{*}$. Some natural problems are:

Problem 2.1. A. In Theorem 1.2, is the condition that $\kappa$ be weakly compact necessary?

B. Give $L^{*}$ a "nice" axiomatization.

In Theorem 1.2 we prove actually:

TheOREM 2.2. A. $L^{*}$ satisfies the completeness theorem; that is, for every sentence $\psi \in L^{*}$ we can find (recursively) a recursive set $\Gamma$ of first-order sentences (or even a single sentence) in a richer language such that $\psi$ has a model iff $\Gamma$ has a model.

B. Every L-model has $L^{*}$-elementary extensions of arbitrary large power.

Clearly $L^{*}$ is interpretable in $L_{\kappa^{+}, \kappa^{+}}$(the language with conjuction on $\kappa$ formulas and quantification on $\kappa$ variables), and by Hanf [Ha 1] every $L$ -

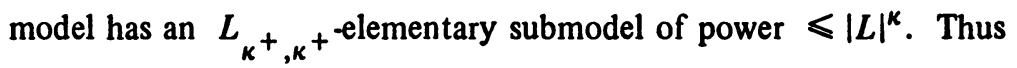

THEOREM 2.3. A. If $|L| \leqslant \lambda=\lambda^{k}$, then every L-model of power $\geqslant \lambda^{k}$ has an $L^{*}$-elementary submodel of power $\lambda^{\kappa}$. (If $|L| \leqslant \kappa$ we can choose $\lambda=2^{k}$.)

B. There is a sentence in $L^{*}$ (having a model) whose models are of power $\geqslant 2^{\kappa}$. There is a consistent theory in $L^{*}$ of power $k$ whose models are of power $\geqslant 2^{k}$. $\left({ }^{1}\right)$

C. Every consistent theory in $L^{*}$ of power $<_{\kappa}$ has a model of power $\leqslant \kappa$.

Proof. A has already been proved.

$B$ is proved by the sentence " $<$ is a linear order, in which every element has immediate predecessor and successor; $\neg\left(Q^{\mathrm{d} c} x, y\right)(x<y) ; P$ is a nonempty convex subset, bounded from above and below, which has no first or last element." Every model of this sentence is of power $\geqslant 2^{\aleph}$.

Let $T$ be the following theory:

(1) " $<$ is a linear order and $7\left(Q^{\mathrm{dc}} x, y\right) x<y$ ".

(2) " $c_{i}<c_{j}$ for all $i<j \in J$ ", where $J$ is a dense $k$-saturated order of power $k$.

Clearly $T$ is consistent. Now let $M \vDash T$ and let $\left(J_{1}, J_{2}\right)$ be a cut of $J$, cf $J_{1}=\mathrm{cf}^{*} J_{2}=\kappa$. So there is an element $a \in M, a_{i}<a<a_{j}$, for all $i \in J_{1}$, $j \in J_{2}$. Thus $\|M\| \geqslant 2^{k}$. This completes the proof of $\mathrm{B}$.

(1) We can improve 2.3B, i.e. there is $\varphi \in L$ which has models only in cardinalities $>\kappa$; see 2.24 . 
C is proved like 1.3 , but we do not need the $P$.

Elimination of the assumption of the existence of a weakly compact cardinal. In place of a weakly compact cardinal we can assume:

(*) There is a proper class of regular cardinals, $C_{1}$, such that for all $\lambda \in$ $C_{1}$ there are $\left\{S_{\alpha}: \alpha<\lambda^{+}, \operatorname{cf} \alpha=\lambda\right\}$ such that for all $S \subseteq \lambda^{+},\left\{\alpha<\lambda^{+}\right.$: cf $\alpha=\lambda, S \cap \alpha=S_{\alpha}$ \} is a stationary set of $\lambda^{+}$.

By Jensen and Kunen [JK, §2, Theorem 1] the class of regular cardinals satisfies $\left({ }^{*}\right)$, if $V=L$.

If $\left({ }^{*}\right)$ holds we can choose $C$ such that $\aleph_{0} \in C\left(\lambda \in C \Rightarrow \lambda^{+} \notin C\right)$, and $C-\left\{\aleph_{0}\right\}$ satisfies $\left(^{*}\right)$.

THEOREM 2.4. If $C$ and $\left({ }^{*}\right)$ are as above, then $L^{*}=L\left(Q_{C}^{\mathrm{cf}}, Q_{C}^{\mathrm{dc}}\right)$ satisfies the compactness theorem.

Proof. The proof is a combination of Keisler [Ke 3, §2] and Chang [Ch 2]. We assume $T$ satisfies the conditions of 1.4 , and every finite subtheory has a model. Choose $\lambda \in C, \lambda \geqslant|T|$ (or even $\lambda \geqslant|T|)$. By $\left({ }^{*}\right)$ clearly $\lambda^{\lambda}=\lambda$. Now we define an increasing elementary sequence of $\lambda$-saturated models $\left\{M_{\alpha}\right\}_{\alpha<\lambda}$, such that for $\alpha<\beta, M_{\beta}$ is an end extension of $M_{\alpha}$, and $M=\bigcup M_{\alpha}$. Also, if $a \in R C^{M}$ then

$$
\begin{aligned}
M \vDash\left(Q_{C}^{c f} x, y\right)(x<y<a) & \Longleftrightarrow \lambda=\operatorname{cf}\{b \in M: b<a\} \\
& \Longleftrightarrow \lambda^{+} \neq \operatorname{cf}\{b \in M: b<a\} ;
\end{aligned}
$$

and if $\left(A_{1}, A_{2}\right)$ is a cut of an order in $M$ which is definable $\left({ }^{2}\right)$ (in $M$ by a formula with parameters) such that $\operatorname{cf} A_{1}=\lambda^{+}$or $\mathrm{cf}^{*} A_{2}=\lambda^{+}$then $A_{1}$ is also definable (in $M$ by a formula with parameters). Clearly $M \vDash T$.

Cofinality quantifiers. We shall deal with logics containing just the generalized quantifier $Q^{\text {cf }}$. We write $Q_{\lambda}^{\text {cf }}$ in place of $Q_{\{\lambda\}}^{\text {cf }}$.

THEOREM 2.5. Let $M$ be an $L$-model of power $>\kappa$. Then $M$ has an $L^{* *}$-elementary submodel of power $\kappa$ where $L^{* *}=L\left(Q_{C_{i}}^{\mathrm{cf}}, Q_{\lambda_{j}}^{\mathrm{cf}}\right)_{i<n, j<\mu}$ if

(1) $\lambda_{j} \leqslant \kappa,|L|+\mu \leqslant \kappa$,

(2) for every $i<n$ there are regular cardinals $\chi_{1}^{i}<\cdots<\chi_{m(i)}^{i}$ such that if for every $l \chi<\chi_{l}^{i} \Longleftrightarrow \chi^{\prime}<\chi_{l}^{i}$ then $\chi \in C_{i} \Longleftrightarrow \chi^{\prime} \in C_{i}$; and

(3) for all regular $\lambda$ there is a regular $\lambda^{\prime} \leqslant k$ such that $\lambda^{\prime} \neq \lambda_{j}$ for all $j$ and $\lambda \in C_{i} \Leftrightarrow \lambda^{\prime} \in C_{i}$.

Proof. The proof is by induction on $\lambda=\|M\|$. As in $\S 1$ we can assume that $|M|$ is an ordinal, say $\lambda+1,<^{M}$ is the order on the ordinals, $R C^{M}$ is the set of regular cardinals in $M, M$ has Skolem functions, and also cofinality

(2) We assume the order is definable. 
Skolem functions (see 1.4(5)). Thus in order that a submodel $N$ of $M$ be an $L^{* *}$-elementary submodel; for all $a \in R C^{N}$ we must have

$$
\begin{aligned}
& M \vDash\left(Q_{\lambda_{j}}^{\mathrm{cf}} x, y\right)(x<y<a) \Longleftrightarrow N \vDash\left(Q_{\lambda_{j}}^{\mathrm{cf}} x, y\right)(x<y<a), \\
& M \vDash\left(Q_{C_{i}}^{\mathrm{cf}} x, y\right)(x<y<a) \Leftrightarrow N \vDash\left(Q_{C_{i}}^{\mathrm{cf}} x, y\right)(x<y<a) .
\end{aligned}
$$

Case 1. $\lambda$ is a regular cardinal: Choose regular $\lambda^{\prime}<\lambda, \lambda^{\prime} \neq \lambda_{j}$ for all $j$, and $\lambda \in C_{i} \Leftrightarrow \lambda^{\prime} \in C_{i}$. Build an increasing sequence $\left\{M_{\alpha}\right\}_{\alpha<\lambda^{\prime}}$ of elementary submodels of $M$ such that

(i) $M_{\alpha} \subsetneq M_{\alpha+1}, M_{\delta}=\bigcup_{\alpha<\delta} M_{\alpha}$ for $\delta$ a limit ordinal, $\left\|M_{0}\right\| \geqslant \kappa$.

(ii) $\left|M_{\alpha}\right|$ is an initial segment of $\lambda$ with the addition of $\lambda$ (which is the last element of $M$ ). $M_{\lambda^{\prime}}$ will be the desired model.

Case 2. $\lambda$ is singular. Choose regular $\chi<\lambda$ such that $\lambda<\chi_{l}^{i} \Leftrightarrow \chi<\chi_{l}^{i}$. There is such a $\chi$ since the number of $\chi_{l}^{i}$ is finite and they are regular thus $\neq \lambda$, and $\lambda$ is a limit cardinal. Let $M_{0}$ be an elementary submodel of $M$ of power $\chi^{\prime}=\chi^{+}+\operatorname{cf} \lambda$ which contains $\left\{\alpha: \alpha \leqslant \chi^{\prime}\right\} \cup\{\lambda\}$. Define by induction on $\alpha \leqslant \chi^{+}$an increasing sequence of elementary submodels of $M,\left\{M_{\alpha}\right\}_{\alpha<\chi^{+}}$, such that $\left\|M_{\alpha}\right\|=\chi^{\prime}, M_{\delta}=\bigcup_{i<\delta} M_{i}$ for $\delta$ a limit ordinal, and if $a \in R C^{M}, \chi<a$, then there is $a^{\prime}<a, a^{\prime} \in M_{\alpha+1}$, such that for every $b<a$ if $b \in M_{\alpha}$, then $b<a^{\prime}$. Clearly if $a \in R C^{M} \cap\left|M_{\chi^{\prime}}\right|$ then the cofinality of $\left\{b \in M_{\chi^{\prime}}: b<a\right\}$ is either $\chi^{+}$or the cofinality of $\{b \in M: b<a\}$. Thus $M_{\chi^{\prime}}$ is an $L^{* *}$. elementary submodel of $M$.

We may assume now that in the definition of $L^{* *}$ the $C_{i}$ are pairwise disjoint.

THEOREM 2.6. Assume $\mu<\aleph_{0}$ in the definition of $L^{* *}$ in 2.5 .

(A) $L^{* *}$ satisfies the completeness theorem and the compactness theorem (and thus the upward Lowenheim-Skolem theorem).

(B) Let $T$ be a theory in $L\left(Q_{C_{i}}^{\text {cf }}, Q_{\lambda_{j}}^{\text {cf }}\right)$. By substituting $\lambda_{j}^{\prime}$ for $\lambda_{j}$ and $C_{i}^{\prime}$ for $C_{i}$ we get a theory $T^{\prime} . T$ has a model iff $T^{\prime}$ has a model, on condition that:

(1) $\lambda_{j_{1}}=\lambda_{j_{2}} \Leftrightarrow \lambda_{j_{1}}^{\prime}=\lambda_{j_{2}}^{\prime}$,

(2) $\lambda_{j} \in C_{i} \stackrel{\Longleftrightarrow}{\Leftrightarrow} \lambda_{j}^{\prime} \in C_{i}^{\prime}$,

(3) if $C_{i}=\left\{\lambda_{j_{l}}: l<l_{0}\right\}$ then $C_{i}^{\prime}=\left\{\lambda_{j_{l}}^{\prime}: l<l_{0}\right\}$.

REMARK. In the completeness theorem we consider a single sentence and the set of quantifiers appearing in it, so there is no need for $\mu<\aleph_{0}$.

SKETCH OF PROOF. Let $T$ be a theory in $L^{* *}$. Without loss of generality $T$ has Skolem functions, there is a symbol $<$ which is an order on the universe, $R C$ is a unary predicate, there are cofinality Skolem functions (see 1.4(5)), and every formula is equivalent to an atomic formula. By adding cofinality quantifiers 
we can assume that $L^{* *}=L\left(Q_{C_{i}}^{\mathrm{cf}}\right)_{i \leqslant n}$ where the $C_{i}$ are disjoint intervals of regular cardinals, $C_{n}=\left\{\lambda: \lambda_{0} \leqslant \lambda\right.$ regular $\} ; \bigcup_{i} C_{i}$ is all the regular cardinals. By using the previous theorem and the set of sentences from Shelah [Sh 2, §4], we get: if every finite $t \subseteq T$ has a model, then $T \cap L$ has a model $M$ for which if $(\forall \bar{x})\left[R^{i}(z) \equiv\left(\bar{Q}_{C_{i}}^{\mathrm{cf}} x, y\right)(x<y<z)\right] \in T$ and $M \vDash R^{i}(z) \wedge R C[z]$, then $\operatorname{cf}\{a: a<z\}=\lambda^{i} .\left(^{3}\right)$ From here, by [Sh 2, §4], the theorem is immediate.

Problem 2.7. When in general is $L^{* *}$ compact?

REMARK. If there is a $C_{i}$ which is an infinite set of $\lambda_{j}$ 's then $L^{* *}$ is not compact. On the other hand, by the previous theorem and ultraproducts, if every finite $t \subseteq T$ has a model, then there is a $T^{\prime}$, as in (B) of the previous theorem, which has a model.

Problem 2.8. Give a nice axiomatization of $L^{* *}$. In one case we have

THEOREM 2.9. If $C \neq \varnothing$, and $C$ is not the class of all regular cardinals, then the following system of axioms is complete for $L\left(Q_{C}^{\mathrm{cf}}\right)$ :

(1) The usual schemes for the first order calculus.

(2) The following scheme (in which variables serving as parameters are not explicitly mentioned):

$\left(Q_{C}^{\mathrm{cf}} x, y\right) \varphi(x, y) \rightarrow[\varphi(x, y)$ is a linear order on $\{y:(\exists x) \varphi\}$

without last element]

$\left(Q_{C}^{\mathrm{cf}} x, y\right) \varphi(x, y) \wedge \neg\left(Q_{C}^{\mathrm{cf}} x, y\right) \psi(x, y) \wedge[\psi(x, y)$ is a linear order

on $\{y:(\exists x) \psi\}$ without last element $]$

$$
\begin{gathered}
\wedge(\forall x, y)\left[\theta(x, y) \rightarrow\left(\exists x_{1}\right) \varphi\left(x_{1}, x\right) \wedge\left(\exists y_{1}\right) \psi\left(y_{1}, y\right)\right] \\
\wedge(\forall y)\left[\left(\exists y_{1}\right) \psi\left(y_{1}, y\right) \rightarrow(\exists x) \theta(x, y)\right] \rightarrow \\
\neg\left[( \forall x _ { 0 } ) ( \exists y _ { 0 } ) \left((\exists x) \varphi\left(x_{1} x_{0}\right) \rightarrow(\exists y) \psi\left(y, y_{0}\right)\right.\right. \\
\left.\left.\wedge\left(\forall x_{1}, y_{1}\right)\left(\psi\left(y_{0}, y_{1}\right) \wedge \theta\left(x_{1}, y_{1}\right) \rightarrow \varphi\left(x_{0}, x_{1}\right)\right)\right)\right]
\end{gathered}
$$

Proof. By the previous theorem it is sufficient to prove that if $T \subseteq L\left(Q_{C}^{\text {cf }}\right)$ is countable, complete, and consistent (by the above axiomatization), then $T$ has a model where we interpret $C$ as $\left\{\boldsymbol{\aleph}_{0}\right\}$ for example. The proof is like [KM].

A quantifier close to the quantifiers we have discussed is

DEFINITION 2.1. $\left(Q^{\mathrm{ec}} x, y\right)[\varphi(x, y), \psi(x, y)]$, which means that the orders defined by $\varphi(x, y)$ and $\psi(x, y)$ on $\{y:(\exists x) \varphi(x, y)\}$ and $\{y:(\exists x) \psi(x, y)\}$, respectively, have the same cofinality.

Conjecture 2.10. The logic $L\left(Q^{\mathrm{ec}}\right)$ is compact and complete (and even has an axiomatization parallel to that of the last theorem). It is not hard to see that

(3) The $\lambda^{i}$,s are arbitrary. 
THEOREM 2.11. (1) There is $\psi \in L\left(Q^{\text {ec }}\right)$ which has a model of power $\aleph_{\alpha}$ iff $\aleph_{\alpha}=\alpha$.

(2) If $\|M\|=\kappa$ where $\kappa$ is a Mahlo number of rank $\alpha+1$, then $M$ has an $L\left(Q^{\text {ec }}\right)$ elementary submodel of power $\lambda$ for some Mahlo number $\lambda<k$ of rank $\alpha$ (actually the set of such $\lambda$ 's which corresponds to $M$ is a stationary set). (For information about Mahlo numbers see Lévy [Le 1].)

(3) If $\kappa$ is not a Mahlo number then there is a model of power $\kappa$, with a finite number of relations, which has no $L\left(Q^{\mathrm{ec}}\right)$-elementary submodel of smaller power.

Generalized second-order quantifiers. Henkin [Hn 1] defined first-order generalized quantifiers as follows: The truth value of $(Q x) \varphi(x)$ in a model $M$ is dependent only on the isomorphism type of $(|M|,\{x: \varphi(x)\})$, i.e., on the powers of $\{x: \varphi(x)\}$ and $\{x: \neg \varphi(x)\}$. This is how the quantifier $\left(Q_{\lambda}^{\mathrm{cr}} x\right) \varphi(x)$ $\Longleftrightarrow|\{x: \varphi(x)\}| \geqslant \lambda$ was reached.

Similarly we may define "generalized second-order quantifier" to be such that the truth value of $(Q P) \varphi(P)$ in $M$ is dependent only on the isomorphism type of $(|M|,\{P: \varphi(P)\})$, like [Li 1].

The regular second-order quantifier is too strong from the point of view of model theory, and so there are no nice model theoretic theorems about it. But there could be generalized second-order quantifiers which are weak enough for their model theory to be nice, for example by satisfying Lowenheim-Skolem, compactness or completeness theorems. In fact the cofinality quantifiers we discussed previously are an example.

DEFINITION 2.2. If $<$ is a linear order on $A$ then an initial segement of $A$ is a set $B \subseteq A$ such that $b<a, a \in B \rightarrow b \in B$. An increasing sequence $\left\{B_{\alpha}: \alpha<\lambda\right\}$ of initial segments is unbounded if every initial segment of $A$ is contained in some $B_{\alpha}$, and it is closed if $B_{\delta}=\bigcup_{\alpha<\delta} B_{\alpha}$ for all limit ordinals $\delta$.

If $\operatorname{cf} A>\omega$ then the closed and unbounded sequences of initial segments of $A$ generate a (nonprincipal) filter $D(A)$ on the set of all initial segments of $A, H(A)$.

Now we define some generalized second-order quantifiers.

DEFinition 2.3. Let $C$ be a class of regular cardinals $>\aleph_{0}$.

$$
\left(Q_{C}^{\text {st }} P, x, y\right)[\varphi(x, y), \psi(P)] \Leftrightarrow\left(Q_{C}^{\text {cf }} x, y\right) \varphi(x, y) \text { and }
$$

if $A=\{y:(\exists x) \varphi(x, y)\}$ then $H(A)-\{P: \psi(P), P \in H(A)\} \notin D(A)$; that is, the above set is stationary.

DEFinition 2.4. Let $\lambda>\aleph_{0}$ be regular, and let $C \subseteq \lambda$. 


$$
\left(Q_{\lambda, C}^{\mathrm{st}}, P, x, y\right)[\varphi(x, y), \psi(P)] \Longleftrightarrow\left(Q_{\lambda}^{\mathrm{st}} P, x, y\right)[\varphi(x, y), \psi(P)] \text { and }
$$

there is a sequence $\left\{P_{i}\right\}_{i<\lambda}$ of initial segments of $\{y:(\exists x) \varphi(x, y)\}$ which is closed and unbounded, and $\left\{i<\lambda: \psi\left(P_{i}\right)\right\} \cup(\lambda-C) \in D(\lambda) .\left({ }^{4}\right)$

REmarK. It is not difficult to see that the above is well defined, for if $\left\{P_{i}^{\prime}\right\}_{i<\lambda}$ is another example of such a sequence $\left\{i: P_{i}=P_{i}^{\prime}\right\} \in D(\lambda)$.

In another example we use a filter similar to that of Kueker [Ku 1]:

For a regular power $\lambda>\aleph_{0}$ and set $A,|A| \geqslant \lambda$, let $S_{\lambda}(A)=\{B: B \subseteq$ $A,|B|<\lambda\}$. $D_{\lambda}(A)$ will be the filter on $S_{\lambda}(A)$ generated by the families $S \subseteq S_{\lambda}(A)$ satisfying

(1) for all $B \in S_{\lambda}(A)$ there is $B^{\prime} \in S$ such that $B \subseteq B^{\prime}$, and

(2) $S$ is closed under increasing unions of length $<\lambda$.

Thus for example if $M$ is a model $\|M\|>\lambda$ whose language is of power $<\lambda$ then $\{|N|: N<M,\|N\|<\lambda\} \in D_{\lambda}(|M|)$.

We can define a suitable quantifier:

Definition 2.5. $\quad\left(Q_{\lambda}^{\text {ss } P}, x\right)[\varphi(x), \psi(P)] \Longleftrightarrow S_{\lambda}(A)-\{P:|P|<\lambda, P \subseteq A \vDash$ $\psi[P]\} \notin D_{\lambda}(A)$ where $A=\{x: \varphi(x)\}$.

Again it is not hard to check that the definition is valid.

Problem 2.12. Investigate the logics with the quantifiers (A) $Q_{\lambda, A}^{\text {st }}$; (B) $Q_{C}^{\text {st }}$; (C) $Q_{\lambda}^{\text {ss }}$. In particular in regard to (1) compactness theorems; (2) downward Lowenheim-Skolem theorems; (3) and transfer theorems (from one $\lambda$ to another). If necessary use $V=L$.

We now mention several partial results in this context.

THEOREM 2.13. (A) If $\|M\|=\kappa, \kappa$ weakly compact, $|L(M)|<\kappa, C$ is the class of all regular cardinals $>\aleph_{0}$ then $M$ has an $L\left(Q_{C}^{\text {st }}\right)$-elementary submodel of smaller power.

(B) $(V=L$.) If $k$ is not weakly compact, then there is a model of power $\kappa$, whose language is countable, which has no proper $L\left(Q_{C}^{\text {st }}\right)$-elementary submodel. (C as above.)

Proof. (A) follows from well-known theorems in set theory.

(B) We shall prove it for regular $k$; the result for a singular one follows from it.

By Jensen [Je 1] there is a set $S$ of ordinals $<\kappa$ of cofinality $\omega$ such that $\kappa-S \notin D(\kappa)$ but for all $\alpha<\kappa$ of cofinality $>\omega, \alpha-\alpha \cap S \in D(\alpha)$. Let $f$ be a two-place function such that for all $\alpha$ of cofinality $\omega\{f(\alpha, n)$ : $n<\omega\}$ is an increasing sequence with limit $\alpha$. We shall choose our model to be $M=(\kappa, S, f,<, \cdots, n, \cdots)$. Assume that $N$ is an $L\left(Q_{C}^{\text {st }}\right)$-elementary submodel of $M$ of smaller power. Let $\alpha=\sup \{\beta: \beta \in N\}$, then $\operatorname{cf} \alpha>\omega$ as

(4) $Q_{\lambda}^{\text {st }}$ means $Q_{\{\lambda\}}^{\text {st }}$ 


$$
M \vDash\left(Q_{C}^{\text {st }} P, x, y\right)(x<y,(\exists z)(\forall v)[P(v) \equiv v<z \wedge S(z)]) \text {, and }
$$

there is a closed and unbounded set $A=\left\{a_{i}: i<\operatorname{cf} \alpha\right\} \subseteq \alpha$ of type cf $\alpha$ which is disjoint with $S$ because $\alpha<\kappa, \operatorname{cf} \alpha>\omega$. For every $a_{i} \in A$, let $a_{i}^{\prime}=$ $\inf \left\{b \in N: b \geqslant a_{i}\right\}$ and $A^{\prime}=\left\{a_{i}^{\prime}: a_{i} \in A\right\}$. Clearly in $N a_{\delta}^{\prime}=\sup \left\{a_{i}^{\prime}: i<\delta\right\}$ for $\delta$ a limit ordinal. Thus $A^{\prime}$ is closed and unbounded in $N$. If $a_{i}^{\prime} \in S$, $\operatorname{cf}\left(a_{i}^{\prime}\right)=\omega$ and so the $f\left(a_{i}^{\prime}, n\right) \in N$ converge to $a_{i}^{\prime}$. So $a_{i}=a_{i}^{\prime}$, contradiction to the disjointness of $A$ and $S$. Thus we have

$$
N \vDash\urcorner\left(Q_{C}^{\text {st }} P, x, y\right)[x<y,(\exists z)(\forall v)(P(v) \equiv v<z \wedge S(z))],
$$

a contradiction.

In regard to the possibility that $N$ be of power $\kappa$, by Keisler and Rowbottom [KR] (see [CK]) we can expand $M$ such that $M$ will be a Jonsson algebra, and that will be a contradiction. If we restrict ourselves to $\aleph_{1}$ we can get stronger results.

THEOREM 2.14. (A) $L\left(Q_{\aleph_{1}}^{\text {cr }}, Q_{\aleph_{1}}^{\text {st }}, Q_{\aleph_{1}, A_{i}}^{\text {st }}\right)_{i<n}$ is $\aleph_{0}$-compact and complete. The consistency of a sentence is just dependent on the Boolean algebra generated by $A_{i} / D\left(\aleph_{1}\right)$, and not on the particular $A_{i} \cdot\left({ }^{5}\right)$

(B) $L\left(Q_{\aleph_{1}}^{\text {st }}, Q_{\aleph_{1}, A_{i}}^{\text {st }}\right)_{i<n}$ is $\aleph_{1}$-compact.

(C) If $T$ is a theory in $L\left(Q_{\lambda}^{\mathrm{cr}}, Q_{\lambda}^{\mathrm{st}}, Q_{\lambda, B_{i}}^{\mathrm{st}}\right)_{i<n}$ and $T^{\prime}$ is the corresponding theory in $L\left(Q_{\aleph_{1}}^{\mathrm{cr}}, Q_{\aleph_{1}}^{\text {st }}, Q_{\aleph_{1}, A_{i}}^{\text {st }}\right)_{i<n}$, and $\left\{B_{i}\right\}$ a partition of $\lambda,\left\{A_{i}\right\}$ a partition of $\omega_{1}, \aleph_{1}-A_{i} \notin D_{i}\left(\aleph_{1}\right), \lambda-B_{i} \notin D(\lambda)$ then $T$ has a model $\Rightarrow T^{\prime}$ has a model.

Proof. (A) Without loss of generality we shall deal with models of power $\aleph_{1}$ whose universe sets are $\omega_{1}$.

It is not-difficult to define a language $L_{1},\left|L_{1}\right| \leqslant|L|$ such that every $L$ model $M,|M|=\omega_{1}$ can be expanded to an $L_{1}$-model $M_{1}$ such that

(1) $M_{1}$ has Skolem functions (dependent only on the formula and not on $M$ ), and every formula (including sentences) is equivalent to an atomic formula,

(2) $<$ is the order on the ordinals, and

(3) $P_{i}^{M}{ }_{1}=A_{i}$.

Let $T$ be a theory in the logic from (A) such that every finite $t \subseteq T$ has a model $M^{t}$. Let $T_{1}$ be the set of sentences of $L_{1}$ holding in $M_{1}^{t}$ for $t$ large enough. Define an increasing elementary sequence of countable $L_{1}$-models: $N_{0}$ will be any countable model of $T_{1}, N_{\delta}=\bigcup_{\alpha<\delta} N_{\alpha}$ for $\delta<\omega_{1}$ limit. If $N_{\alpha}$ is defined $N_{\alpha+1}$ will be an end extension of $N_{\alpha}$ (i.e. $N_{\alpha+1} \vDash a<b \in$ $\left.N_{\alpha} \rightarrow a \in N_{\alpha}\right)$ such that there is a first element $a_{\alpha}$ in $\left|N_{\alpha+1}\right|-\left|N_{\alpha}\right|$ and $a_{\alpha} \in P_{i} \Longleftrightarrow \alpha \in A_{i}$. The proof that this is possible is similar to Keisler [Ke 2],

$(5)$ Of course, every model with language $L$ has an elementary submodel of cardinality $\leqslant|L|+\aleph_{1}$ in this logic. 
[Ke 3]. It is not difficult to check that $U_{\alpha<\omega_{1}} N_{\alpha}$ is the required model of $T$.

The proof of the completeness is similar, but $T_{1}$ must be defined more carefully.

(B) The proof is similar to that of (A); here $N_{\alpha+1}$ will be an expansion (as well as an extension) and instead of the demand that $N_{\alpha+1}$ be an end extension, we only need that for all $\delta \leqslant \alpha$ limit ordinal the type $\left\{a_{i}<x: i<\delta\right\} \cup$ $\left\{x<a_{\delta}\right\}$ be omitted. $\left.{ }^{6}\right)$

(C) The proof is similar.

The class $K_{\lambda}$. After the proof of the previous theorem it is natural to consider the following class of models which is somewhat parallel to the class of $\kappa$-like models.

DEFINITION 2.6. Let $\lambda$ be regular. $M \in K_{\lambda}$ iff $<$ linearly orders $\{x: M \vDash(\exists y)(x<y \vee y<x)\}$ with cofinality $\lambda$, and there is a continuous increasing unbounded sequence $\left\{a_{i}\right\}_{i<\lambda}$ (i.e. for all $\delta<\lambda$ limit, the type $\left\{a_{i}<x<a_{\delta}: i<\delta\right\}$ is omitted by $M$ ).

From the previous theorem follows

THEOREM 2.15. If $|T| \leqslant \aleph_{1}$ ( $T$ a first-order theory) and every finite $t \subseteq T$ has a model in some $K_{\lambda}, \lambda>\aleph_{0}$ then $T$ has a model in $K_{\aleph_{1}}$.

It is easily proven that

THEOREM 2.16. If $M \in K_{\lambda}, \mu<\lambda$ regular, $|L(M)|<\lambda$ then $M$ has an elementary submodel in $K_{\mu}$.

Somewhat less immediate is the following.

THEOREM 2.17. (A) If for every $n<\omega$ every finite $t \subseteq T$ has a model in some $K_{\lambda}$ for $\lambda \geqslant \aleph_{n}$, then $T$ has a model in $K_{\lambda}$ for all $\lambda$.

(B) (Completeness.) The set of sentences true in every model of $K_{\aleph_{\omega+1}}$ is recursively enumerable.

Proof. Without loss of generality assume that $T$ has Skolem functions. For every ordinal $\alpha$ define

$$
\begin{aligned}
\Sigma_{\alpha}=\left\{\tau\left(y_{i_{1}}, \cdots, y_{i_{n}}\right)<y_{i_{(k+1)}} \rightarrow\right. & \tau\left(y_{i_{1}}, \cdots, y_{i_{n}}\right)<y_{\left(i_{k}+1\right)}: \\
& \left.\tau \text { is a term of } L(T), i_{1}<\cdots<i_{n}<\alpha\right\} .
\end{aligned}
$$

It is clear that: $T \cup \Sigma_{n}$ is consistent for all $n \Leftrightarrow T \cup \Sigma_{\alpha}$ is consistent for all $\alpha \Leftrightarrow$ for all $\lambda T$ has a model in $K_{\lambda}$; for if $M$ is a model of $T \cup \Sigma_{\lambda}$

(6) We should first assume w.l.o.g. that our language $L$ has a countable sublanguage $L_{1}$, such that $L-L_{1}$ consist of individual constants $\left\{c_{i}: i<\omega_{1}\right\}, P\left(c_{i}\right) \in T$; and every finite $t \subset T$ has a model $M^{t},\left|M^{t}\right|=\omega_{1}, P^{M^{t}}$ is finite, and in $M_{1}^{t}$, every limit ordinal is the universe of a submodel of $M_{1}^{t}$, and (1)-(3) from the proof of (A) holds. 
which is the closure of $\left\{y_{i}: i<\lambda\right\}$ under Skolem functions, then $M \in K_{\lambda}$. Thus it is sufficient to prove:

(*) For all $n$ and all finite $\Sigma_{n}^{\prime} \subseteq \Sigma_{n}$ and all $M \in K_{\aleph_{n}}$ there are $y_{0}, \cdots, y_{n-1} \in M$ satisfying $\Sigma_{n}^{\prime}$.

We shall show by downward induction on $m<n$ that:

(**) There are

(1) $y_{m+1}<\cdots<y_{n-1}$ (when $m=n-1$ this is an empty sequence).

(2) $a_{j}^{m}<a_{i}^{m}<y_{m+1}$ for all $j<i \leqslant \aleph_{n-m}, a_{\aleph_{n-m}^{m}}^{m}=y_{m+1}$ (except when $n=m$ ).

(3) For all $\delta \leqslant \aleph_{n-m}$ limit ordinal there is no $x$ such that $a_{i}^{m}<x<$ $a_{\delta}^{m}$ for all $i<\delta$.

(4) If $\tau$ occurs in $\Sigma_{n}^{\prime}, b_{1}, \cdots, b_{k} \in\left\{a_{i}^{m}: i<\alpha<\aleph_{n-m}\right\} \cup\left\{y_{m+1}\right.$, $\left.\cdots, y_{n-1}\right\}$, then $M \vDash \tau\left(b_{1}, \cdots, b_{k}\right)<y_{m+1} \rightarrow \tau\left(b_{1}, \cdots, b_{k}\right)<a_{\alpha+1}^{m}$ (if $m=n-1$ we have instead $M \vDash \tau\left(b_{1}, \cdots, b_{k}\right)<a_{\alpha+1}^{m}$ ).

(5) $y_{m+1}, \cdots, y_{n}$ satisfy the corresponding formulas of $\Sigma_{n}^{\prime}$. Now for $m=n$ choose an increasing unbounded continuous sequence $\left\{a_{i}^{n}\right\}_{i<\aleph_{n}}$.

Assume that we have already completed stage $m+1$, and we shall define for $m$ (for simplicity let $m<n$ ) there is a closed unbounded set $S \subseteq\{\alpha$ : $\left.\alpha<\aleph_{m+1}\right\}$ such that for $\alpha \in S, \sigma_{1}, \cdots, \sigma_{l} \in\left\{a_{i}^{m+1}: i<\alpha\right\} \cup\left\{y_{i}: m<\right.$ $i<n\}$, and $\tau$ which occurs in $\Sigma_{n}^{\prime}$ we have $\tau\left(\sigma_{1}, \cdots, \sigma_{l}\right)<a_{\aleph_{n-m}}^{m+1} \rightarrow$ $\tau\left(\sigma_{1}, \cdots, \sigma_{l}\right)<a_{\alpha}^{m+1}$. Choose $\alpha_{0} \in S$ such that $\operatorname{cf}(S \cap \alpha)=\aleph_{m}^{n-m}$ and define $y_{m}=a_{\alpha}^{m+1}$. Let $\left\{\alpha_{i}: i<\aleph_{m}\right\}$ be an increasing unbounded continuous sequence in $S \cap \alpha$ (it is easy to verify that there is such a sequence), and let $a_{i}^{m}=a_{\alpha_{i}}^{m+1}$. (If $m=0$ there is no need to choose $a_{i}^{m}$, and thus it was sufficient to assume that $M \in K_{\kappa_{n-1}}$.)

THEOREM 2.18. For all $n<\omega$ there is a sentence $\psi_{n}$ having a model in $K_{\aleph_{n}}$ but no model in $K_{\aleph_{n+1}}$.

Proof. $\psi_{n}$ will more or less characterize $\left(\omega_{n},<\right)$.

$\psi_{0}$ will say that there is a first element, every element has a successor, and every element (except the first) has a predecessor.

$\psi_{n+1}$ will say that $\left\{a: a<c_{i}\right\}$ satisfies $\psi_{i}$ for $i \leqslant n$ ( $c_{i}$ being an individual constant), $P_{0}, \cdots, P_{n}$ is a partition of the limit elements, and if $a \in P_{i}$ then $\left\langle F_{i}(a, x): x\left\langle c_{i}\right\rangle\right.$ is an increasing, continuous, unbounded sequence in $\{y: y<a\}$.

Similar theorems may be proved with omitting types as in [Mo 1]. For example if $T$ is countable and has a model in $K_{\aleph_{\omega_{1}}}$ omitting a type $p$, then
for all $\lambda T$ has a model in $K_{\lambda}$ omitting $p$.

Problem 2.19. Prove the compactness of $K_{\aleph_{n}}$, for $1<n<\omega$. 
REMARK. If we relax the condition of continuity at $\delta$ of cofinality $\omega$ then we can prove this as in [Sh 1]. Since then the class is closed under ultraproducts of $\aleph_{0}$ models. In general it suffices to prove the $\aleph_{0}$-compactness of $K_{\aleph_{n}}$.

General questions. A general problem (which is of course not new) about abstract logic is

Problem 2.20. Find the logical connections between the following properties of the abstract logic $L^{*}$ :

(A) $L^{*}$ is first-order logic.

(B) ${ }_{\lambda} L^{*}$ satisfies the compactness theorem for theories of power $\leqslant \lambda$.

(C) $=(B)_{\infty} L^{*}$ satisfies the compactness theorem.

(D) $L^{*}$ satisfies the $\lambda$-downward Lowenheim-Skolem theorem. (If $\psi \in$ $L^{*}$ has a model then $\psi$ has a model of power $\leqslant \lambda$.)

(E) $L^{*}$ satisfies the $\lambda$-upward Lowenheim-Skolem theorem. (If $\psi$ has a model of power $\geqslant \lambda$, then $\psi$ has a model of arbitrarily large power.)

(F) $L^{*}$ satisfies Craig's theorem.

(G) $L^{*}$ satisfies Beth's theorem.

(H) $L^{*}$ satisfies the Feferman-Vaught theorems for

(1) Sum of models.

(2) Produst of models.

(3) Generalized product of models.

(I) $L^{*}$ satisfies the completeness theorem (assuming that the set of sentences is recursive in the language).

It is known that (A) implies the others; for $\mu<\lambda(\mathrm{C}) \rightarrow(\mathrm{B})_{\lambda} \rightarrow(\mathrm{B})_{\mu}$, $(\mathrm{E})_{\mu} \rightarrow\left(\mathrm{E}_{\lambda}\right),(\mathrm{D})_{\mu} \rightarrow(\mathrm{D})_{\lambda} ;(\mathrm{F}) \rightarrow(\mathrm{G}),(\mathrm{C}) \rightarrow(\mathrm{E})_{\aleph_{0}},(\mathrm{H})(3) \rightarrow(\mathrm{H})(2) \rightarrow$ (H)(1). Lindenström [Li 1], [Li 2] proved (and Friedman [F 1] reproved). $(B)_{\aleph_{0}} \wedge(D)_{\aleph_{0}} \rightarrow(A),(E)_{\aleph_{0}} \wedge(D)_{\aleph_{0}} \rightarrow(A),(F) \wedge(D)_{\aleph_{0}} \rightarrow(A)$. The method of proof is by encoding Ehrenfeucht-Fraisse games.

Special questions which look interesting to me are

Problem 2.21. Is there a logic $L^{*}$ stronger than first-order logic which is $\aleph_{0}$-compact and satisfies Craig's theorem? Do sums of models preserve elementary equivalence for $L^{*}$ ?

Is there an expansion of $L\left(Q_{\aleph_{1}}^{\text {cr }}\right)$ satisfying this? Keisler and Silver showed that $L\left(Q_{\lambda}^{\text {cr }}\right)$ does not satisfy Craig's theorem. Friedman [Fr 2] showed that Beth's theorem is also not satisfied. Similarly it is not hard to show that all the logics with the quantifiers $Q^{\mathrm{cf}}, Q^{\mathrm{dc}}, Q^{\mathrm{cc}}, Q^{\text {st }}$ (all or some of them) do not satisfy Craig's theorem, but satisfy $(H)(1) . Q^{s s}$ does not satisfy (H)(1).

Problem 2.22. Does $L\left(Q_{\aleph_{1}}^{s s}\right)$ satisfy Craig's theorem, if we restrict ourselves to models of power $\leqslant \aleph_{1}$ ? 
Problem 2.23. Find a natural characterization for $L\left(Q_{\aleph_{1}}^{\mathrm{cr}}\right)$. (For $L_{\infty, \omega}$, $L_{\omega_{1}, \omega}$, etc. Barwise [Ba 1] found one.)

LEMMA 2.24. Let $Q^{1}$ be the quantifier $Q_{\aleph_{0}}^{\mathrm{dc}}$; there is a sentence $\psi$ in $L\left(Q^{1}\right)$, which has only well-ordered models, and has a model of order type a for every $\alpha \geqslant 2^{\aleph_{0}}$. (Thus $L\left(Q^{1}\right)$ is not compact.)

Proof. Let $\psi_{1}$ say:

1. $P_{1}, P_{2}, P_{3}, P_{4}$ (one place predicates) are a partition of the universe.

2 . $\leqslant$ is a total order of the universe, $S$ is the successor function in $P_{1}$ and $P_{2}$ (so $P_{1}$ and $P_{2}$ are closed under $S$ ) and each $P_{i}$ is a convex subset.

3. $F$ is a one place function mapping $\boldsymbol{P}_{\mathbf{3}}$ into $\boldsymbol{P}_{\mathbf{2}}$.

4. $G$ is a two-place function from $P_{3}$ to $P_{1}$ and $\left(\forall x \in P_{3}\right)\left(\forall y \in P_{3}\right)\left(\forall z \in P_{1}\right)\left[S(z) \leqslant G(x, y) \wedge x<y \equiv\left(\forall v \in P_{2}\right)\left(\exists x^{\prime}, y^{\prime} \in P_{3}\right)\right.$

$$
\left.\left(x<x^{\prime}<y^{\prime}<y \wedge \varphi\left(x^{\prime}, y^{\prime}, v\right) \wedge z \leqslant G\left(x^{\prime}, y^{\prime}\right)\right)\right]
$$

where $\varphi(x, y, z)=P_{3}(x) \wedge P_{3}(y) \wedge P_{2}(z) \wedge x<y \wedge(\forall v)(x<v<y \rightarrow z<F(v))$.

5. $\left(\forall z \in P_{1}\right)\left(\exists x, y \in P_{3}\right)(x<y \wedge G(x, y)=z)$.

6. The cofinality of $P_{2}$ is $\aleph_{0}$ (just say $F$ is an anti-isomorphism from $\left(P_{2}, \preccurlyeq\right)$ onto $\left(P_{4}, \leqslant\right)$, and

$$
\begin{gathered}
\left(Q^{1} x y\right)\left[\left(P_{2}(x) \vee P_{4}(x)\right) \wedge\left(P_{2}(y) \vee P_{4}(y)\right) \wedge x<y\right] \\
\wedge\left(Q^{1} x y\right)\left(P_{2}(x) \wedge P_{2}(y) \wedge x<y\right)
\end{gathered}
$$

7. $\left(Q^{1} x y\right)\left(P_{3}(x) \wedge P_{3}(y) \wedge x<y\right)$.

Suppose $M \vDash \psi_{1}$ and $c_{n}$ is a strictly decreasing sequence in $P_{1}^{M}$; let $d_{n}(n<\omega)$ be an increasing unbounded sequence in $P_{2}^{M}$, and define inductively $x_{n}, y_{n} \in P_{3}^{M}, x_{n}<x_{n+1}<y_{n+1}<y_{n}$, and $G\left(x_{n}, y_{n}\right) \geqslant c_{n}$, and $\varphi\left(x_{n+1}, y_{n+1}, d_{n}\right)$. For $n=0$ use 5 , for $n+1$ use 4 . So by $\varphi$ 's definition for no $z, x_{n}<z<y_{n}$ for every $n$ (as then $F(z)$ cannot be defined); contradicting 7). So in every model of $\psi_{1}, P_{1}$ is well-ordered. Now we define by induction on $\alpha$ orders $I_{\alpha}$ and functions $F_{\alpha}: I_{\alpha} \rightarrow \omega$ as follows:

$I_{0}$ is $\aleph_{1}$-saturated order of cardinality $2^{\aleph_{0}} ; F_{0}$ is constantly zero.

$I_{\alpha+1}=\left\{\langle i, a\rangle: i \in \omega+1, a \in I_{\alpha}\right\}$ ordered lexicographically.

$F_{\alpha+1}(\langle i, a\rangle)=F(a)+i$ for $i<\omega$, and zero otherwise.

$I_{\delta}=\left\{\langle\alpha, a\rangle: \alpha \leqslant \delta+1, a \in I_{\alpha}\right\}$ ordered lexicographically.

$F_{\delta+1}(\langle\alpha, a\rangle)=F_{\alpha}(a)$ for $\alpha<\delta$, and zero otherwise.

Now we can easily define $M^{\alpha} \vDash \psi_{1}, P_{1}^{M^{\alpha}}=1+\alpha, P_{2}^{M^{\alpha}}=\omega, P_{3}^{M^{\alpha}}=I_{\alpha}, F^{M^{\alpha}} \supset$ $F_{\alpha}, P_{4}^{M^{\alpha}}=\omega^{*}$. The change to $\psi$ is now only technical.

Added in proof. 1. Schmerl, in a preprint "On $\kappa$-like structures which embed stationary and closed unbounded subsets" proved interesting results on problems closely related to $\left(Q_{\lambda}^{\text {st }} x\right)$. 
2. The author proved that a variant of Feferman-Vaught theorem and Beth theorem implies Craig theorem. This and other results will appear.

3. Why do we use $Q_{\left\{\kappa_{0}, \kappa\right\}}^{\mathrm{cf}}, Q_{\left\{\kappa_{0}, \kappa\right\}}^{\mathrm{dc}}$, and not just $Q_{\left\{\kappa_{0}\right\}}^{\mathrm{cf}}, Q_{\left\{\kappa_{0}\right\}}^{\mathrm{dc}}$ in Definition 1.4? (Note that $Q_{\aleph_{0}}^{\text {cf }}$ is added just for convenience.)

\section{REFERENCES}

340.

[Ba 1] K. J. Barwise, A bsolute logics and $L_{\infty, \omega}$, Ann. Math. Logic 4 (1972), 309-

[Ch 1 ] C. C. Chang, Two refinements of Morley's method on omitting types of elements, Notices Amer. Math. Soc. 11 (1964), 679. Abstract \#64T-449.

[Ch 2] - A note on the two cardinal problem, Proc. Amer. Math. Soc. 16 (1965), 1148-1155. MR 33 \#1238.

[CK] C. C. Chang and H. I. Keisler, Model theory, North-Holland, Amsterdam, 1973.

[EM] A. Ehrenfeucht and A. Mostowski, Models of axiomatic theories admitting automorphisms, Fund. Math. 43 (1956), 50-68. MR 18, 863. 1970.

[Fr 1] H. Friedman, Why first order logic, Mimeographed Notes, Stanford University,

[Fr 2] - Beth theorem in cardinality logic, Israel J. Math 14 (1973), 205-212.

[FV] S. Feferman and R. L. Vaught, The first order properties of products of alge. braic systems, Fund. Math. 47 (1959), 57-103. MR 21 \#7171.

[Fu 1 ] G. Fuhrken, Languages with added quantifier "there exist at least $\aleph_{\alpha}$ ", Theory of Models (Proc. 1963 Internat. Sympos. Berkeley), North-Holland, Amsterdam, 1965, pp. 121-131. MR 39 \#2624.

[Ha 1] W. Hanf, Incompactness in languages with infinitely long expressions, Fund. Math. 53 (1963/64), 309-324. MR 28 \#3943.

[He 1] M. Helling, Hanf numbers for some generalizations of first-order languages, Notices Amer. Math. Soc. 11 (1964), 670. Abstract \#64T-448.

[Hn 1] L. Henkin, Some remarks on infinitely long formulas, Infinitistic Methods (Proc. Sympos. Foundations of Math, Warsaw, 1959), Pergamon, Oxford; PWN, Warsaw, 1961, pp. 167-183. MR 26 \#1244.

[Je 1] R. Jensen, The fine structure of the constructible heirarchy, Ann. Math. Logic 4 (1972), 229-308.

[JK] R. Jensen and K. Kunen, Some combinatorial properties of $L$ and $V$ (notes).

[Ke 1] H. J. Keisler, Some model theoretic results for w-logics, Israel J. Math. 4 (1966), 249-261. MR 36 \#4974.

[ Ke 2] - Logic with the quantifier "there exist uncountably many", Ann. Math. Logic 1 (1970), 1-93. MR 41 \#8217.

[Ke 3] - Models with tree structures, Proc. Sympos. Pure Math., vol. 25 Amer. Math. Soc., Providence, R. I., 1974, pp. 331-348.

[KM] H. J. Keisler and M. D. Morley, Elementary extensions of models of set theory, Israel J. Math. 6 (1968), 49-65. MR 38 \#5611.

[KR 1] H. J. Keisler and F. Rowbottom, Constructible sets and weakly compact cardinals, Notices Amer. Math. Soc. 12 (1965), 373-374. Abstract \#65T-186.

[Ku 1 ] D. W. Kueker, Löwenheim-Skolem and interpolation theorems in infinitary languages, Bull. Amer. Math. Soc. 78 (1972), 211-215. MR 45 \#36.

[Le 1] A. Lévy, Axiom schemata of strong infinity in axiomatic set theory, Pacific J. Math. 10 (1960), 223-238. MR 23 \#A1522.

[Li 1] P. Lindström, First order predicate logic with generalized quantifiers, Theoria 32 (1966), 186-195. MR 39 \#5329. 39 \#5330.

[Li 2] On extensions of elementary logic, Theoria 35 (1969), 1-11. MR

[MA 1 ] M. Magidor and J. I. Malitz, Compact extensions of ${ }_{L} Q$, Notices Amer. Math. Soc. 20 (1973), A-501. Abstract \#73T-E79. 
[MK 1] J. A. Makowsky, On continuous quantifiers, Notices Amer. Math. Soc. 20 (1973), A-502. Abstract \#73T-E84.

[Mo 1] M. D. Morley, Omitting classes of elements, Theory of Models (Proc. 1963 Internat. Sy mpos. Berkeley), North-Holland, Amsterdam, 1965, pp. 265-273. MR 34 \#1189.

[MS 1] A. Mostowski, On a generalization of quantifiers, Fund. Math. 44 (1957), 1236. MR 19, 724.

[MV] M. Morley and R. Vaught, Homogeneous universal models, Math. Scand. 11 (1962), 37-57. MR 27 \#37.

[Si 1] J. Silver, Some applications of model theory in set theory, Ann. Math. Logic 3 (1971), 54-110.

[Sc 1] J. H. Schmerl, An elementary sentence which has ordered models, J. Symbolic Logic 32 (1972), 521-530.

[Sh 1] S. Shelah, Two cardinal compactness, Israel J. Math. 7 (1971), 193-198; see also Notices Amer. Math. Soc. 18 (1971), 425. Abstract \#71T-E15. 247-267.

[Sh 2] - On models with power like orderings, J. Symbolic Logic 37 (1972),

[SS] J. H. Schmerl and S. Shelah, On power-like models for hyperinaccessible cardinals, J. Symbolic Logic 37 (1972), 531-537. MR 47 \#6474.

[TR 1] L. H. Tharp, The uniqueness of elementary logic, Notices Amer. Math. Soc. 20 (1973), A-23. Abstract \#73T-E8.

[Va 1] R. L. Vaught, The Löwenheim-Skolem theorem, Logic, Methodology and Philos. Sci. (Proc. 1964 Internat. Cong.), North-Holland, Amsterdam, 1965, pp. 81-89. MR 35 \#1461. ISRAEL

INSTITUTE OF MATHEMATICS, THE HEBREW UNIVERSITY, JERUSALEM, 